\title{
Hörer- und Sprechertypen in Bremen und Hamburg \\ Eine Untersuchung zu Sprachwissen, Sprachwahrnehmung \\ und Sprachgebrauch
}

Yvonne Hettler (Hamburg)

\begin{abstract}
Regarding the perception of regional speech and the salience of language variants it is to assume, that they are being influenced by individual features of the specific speaker/listener. However, most recent studies dealing with the salience of language phenomena neglect factors like, for example, language awareness or the profession of speakers. This article focuses on the correlation between speech perception, speech production and individual characteristics of speakers like the metalinguistic knowledge they have. It presents selected results of a study dealing with the perception and production of regional speech in Bremen and Hamburg and discusses different types of speaker/listener profiles.
\end{abstract}

\section{$1 \quad$ Einleitung}

Die Auffälligkeit, also Salienz, von Sprachphänomenen ist seit Schirmunskis Einteilung in primäre und sekundäre Merkmale (cf. Schirmunski 1928/29) ein immer wiederkehrendes Thema in der Linguistik, zunächst in Hinblick auf Sprachwandel, später auch verstärkt in einem soziolinguistischen Kontext. In rezenten Studien wird Salienz als ein dynamisches Konstrukt gesehen (cf. u. a. Lenz 2010: 101), das zwar von merkmalsinhärenten Faktoren beeinflusst werden kann, aber auch hörer-, respektive sprecherindividuell ist. Dementsprechend ist anzunehmen, dass die Perzeption regionaler Merkmale u. a. von Faktoren wie dem Einfluss der Erziehung, dem Beruf oder einem besonders ausgeprägten metasprachlichen Wissen bzw. Sprachwissen beeinflusst werden kann. ${ }^{1}$ Bisherige empirische Studien zur Einzelmerkmalssalienz fokussieren jedoch häufig einen quantitativen Ansatz, der zwar Parameter wie die Herkunft der Hörer bei der Thematisierung der Merkmalsperzeption heranzieht (cf. u. a. Elmentaler/Gessinger/Wirrer 2010, Lorenz 2014, Kiesewalter 2014) nicht jedoch unter einem qualitativen Gesichtspunkt bspw. sprachbiografische Faktoren einzelner Gewährspersonen miteinbezieht. ${ }^{2}$ Im vorliegenden Text soll der Fokus deswegen unter einem qualitativ ausgerichteten Gesichtspunkt auf hörer- bzw. sprecherindividuelle Sprachwissensbestände sowie Sprach-

\footnotetext{
${ }^{1} \mathrm{Zu}$ den Termini metasprachliches Wissen bzw. Sprachwissen siehe Kapitel 3.

2 Eine Ausnahme stellt hier u. a. die Publikation von Keuch/Wirrer (2014) dar, die sprecherindividuelle Faktoren wie das Sprachbewusstsein von Gewährspersonen mit deren Perzeption salienter Varianten in Verbindung stellt. Siehe auch Kapitel 2.
}

Linguistik online 85, 6/17 - http://dx.doi.org/10.13092/lo.85.4081

CC by 3.0 
wahrnehmungs- und Sprachgebrauchsmuster von norddeutschen Sprechern gerichtet werden. Hierzu einleitend sollen zunächst salienzbedingende Parameter sowie der Einfluss sprachlicher Wissensbestände auf die Wahrnehmung regionaler Varianten diskutiert sowie hörerindividuelle, auf Sprache bezogene Wissensbestände und deren Erwerb genauer betrachtet werden. Im Folgenden werden ausgewählte Ergebnisse einer Studie zur Wahrnehmung, Bewertung und Realisierung regionaler Merkmale in Bremen und Hamburg präsentiert, ${ }^{3}$ in der durch die Verknüpfung objektiver Daten zum Sprachgebrauch sowie subjektiver Daten zur Wahrnehmung von und dem Wissen über regionale Varianten Sprecher-/Hörertypen gebildet werden.

\section{Salienz und Sprachwissen}

Beschäftigt man sich mit der Wahrnehmung regionaler Varianten oder Varietäten spielt das Konzept der Salienz eine wichtige Rolle. Die Faktoren, die die Auffälligkeit von Merkmalen stützen, sind vielfältig. Die wichtigsten, in der Forschungsliteratur diskutierten, Parameter sind: 4

1. Phonetischer Abstand: Je größer die Distanz des Merkmals zur Standardsprache ist, desto auffälliger ist die Variante (cf. Schirmunski 1928/29: 167). ${ }^{5}$

2. Frequenz: Wenig frequente Merkmale bzw. fremde Varianten sind besonders auffällig (cf. Elementaler/Gessinger/Wirrer 2010: 119 u. Rácz 2012: 62).

3. Sprachebene: Lexikalische sowie morpho-syntaktische Abweichungen von der Standardsprache sind für den Hörer salienter als phonetisch-phonologische, wobei es innerhalb der grammatischen Phänomene Hierarchien zu geben scheint und Elemente, die den Kasusgebrauch betreffen, salienter als jene der Satzstellung sind (cf. Trudgill 1986: 25 u. Mihm 1985: 183f.).

4. Gebrauch: Auffällige (primäre) Merkmale werden eher abgelegt als sekundäre (cf. Schirmunski 1928/29: 168).

5. Bewertung: Stigmatisierte oder sehr prestigehaltige Merkmale sowie sprachliche Stereotypen besitzen eine hohe Salienz und werden, bspw. in den Medien, häufiger metakommuniziert (cf. u. a. Trudgill 1986: 11 u. Lenz 2010: 100f.).

6. (Sprach)biografische bzw. hörerindividuelle Parameter: Individuelle Hörereigenschaften wie das Alter und Geschlecht, die Herkunft, der Beruf, das metasprachliche Wissen oder die sensorische Fähigkeit (cf. u. a. Hettler 2015, Kerswill/Williams 2002, Keuch/Wirrer 2014, Kiesewalter 2014) können eine Auswirkung auf die Perzeption sprachlicher Varianten haben.

\footnotetext{
${ }^{3}$ Teile der hier dargestellten Ergebnisse und theoretischen Ausführungen sind der Dissertation Salienz, Bewertung und Realisierung regionaler Merkmale in Bremen und Hamburg (Hettler 2015) entnommen worden.

${ }^{4}$ Zur Diskussion aktueller Salienzmodelle siehe insbesondere Purschke 2014 und Hettler 2015.

${ }^{5} \mathrm{Zu}$ einem Überblick über die Diskussion von Schirmunskis Kriterien und des problematischen Faktors ,Größe siehe bspw. Lenz 2010.
} 
Die Auffälligkeit von Merkmalen wird also keinesfalls nur von merkmalsintrinsischen Faktoren gesteuert sondern hängt in verstärktem Maße auch von der Bewertung von Varianten und einer Vielzahl außersprachlicher Parameter ab. ${ }^{6}$

Das aktuellste und bis dato umfassendste Salienzmodell von Purschke $(2011,2014)$ gibt einen Überblick über die Prozesse, die bei der Perzeption von Varianten eine Rolle spielen. Unter dem Oberbegriff der Sozio-pragmatischen Indexikalität wird unter Salienz die rein perzeptive Auffälligkeit des Merkmals gesehen, die durch das Salienzpotential beeinflusst werden kann, also merkmalsinhärenten Qualitäten, die Purschke auch als „Prominenz“ bezeichnet (Purschke 2014: 36). Zu der reinen Auffälligkeit des Merkmals kommt die Pertinenz hinzu, unter der die subjektive Relevanz substandardlicher Einheiten, also eine situative Bedeutung, die der Hörer salienten Merkmalen zugesteht (oder nicht) und von der sein kommunikatives Handeln beeinflusst wird, verstanden wird. Sprachliche Merkmale können intersubjektiv unterschiedlich bewertet werden und „ohne kommunikative Konsequenzen bleiben“ (Purschke 2011: 82), z. B. durch eine Veränderung des Sprachverhaltens bzw. der Wahl bestimmter Varianten (cf. Purschke 2011: 84), wenn sie „situativ als nicht signifikant oder interaktionell als akzeptabel bewertet [werden].“ (Purschke 2011: 82). In dem Modell werden also sowohl merkmalsinhärente Faktoren als auch die individuelle Hörerwahrnehmung berücksichtigt.

Für die vorliegende Studie ist vor allem der Einfluss sprachbiografischer und hörerspezifischer Faktoren auf die Wahrnehmung (siehe Punkt 6 der obigen Auflistung), von Interesse sowie, wie der Sprachgebrauch von Sprechern mit ihrer Sprachbewertung- und Sprachwahrnehmung korreliert. ${ }^{7}$ Zum Zusammenhang von hörerindividuellen Faktoren und der Perzeption sprachlicher Merkmale gibt es bisher erst sehr wenige Studien, wenngleich schon Kerswill/Williams konstatieren, dass Salienz u. a. von außersprachlichen Parametern die kognitiver, pragmatischer, sozialpsychologischer oder soziodemografischer Natur sein können, abhängig ist (Kerswill/Williams 2002: 105). Auch Lenz weist auf den Zusammenhang zwischen individuell unterschiedlicher Sprachwahrnehmung und sprachbiografischen Faktoren hin, wenn sie festhält, dass die Intensität des Kontaktes zu anderen Dialektsprechern aus der eigenen oder einer anderen Region die Perzeption beeinflussen kann, da „die explizite Widerspiegelung des eigenen Sprachverhaltens durch Außenstehende [...] die Selbstwahrnehmung [schärft]“ (Lenz 2010: 101). Erste empirische Befunde in Bezug auf das metasprachliche Wissen von Gewährspersonen und deren Wahrnehmung salienter Formen liefern Keuch/Wirrer, die feststellen, dass diejenigen Gewährspersonen, die „über ein

\footnotetext{
${ }^{6}$ Darüber, ob Salienz auch immer mit objektiven Kriterien in Verbindung gebracht werden kann, gibt es Uneinigkeit. So fordert Glauninger (2014: 25) „die Überwindung [des] Postulats“, dass Salienz als „subjektivattitudinales Datum“ mit „objektiven Entitäten/Größen“ korrespondiert. Angesichts der Tatsache, dass es jedoch durchaus Merkmale gibt, die von fast allen Sprechern als salient empfunden werden (cf. Hetter 2015 u. Kapitel 4 dieses Textes), scheint es fraglich, ob das Vorherrschen „objektiver“ Kriterien, wie bestimmter merkmalsintrinsischer Faktoren, tatsächlich immer außer Acht gelassen werden kann.

${ }^{7}$ Semiotische Aspekte von Salienz, wie sie z. B. Glauninger (2014) betont und die situative Bedeutung, die der Hörer in der (Alltags)kommunikation salienten Merkmalen zugesteht und von der sein kommunikatives Handeln beeinflusst wird (cf. Purschke 2011), werden in der vorliegenden Studie und dem zugrunde liegenden Untersuchungsaufbau dagegen nicht fokussiert. Der Einfluss der speziellen Test- und Interviewsituation bzw. der Interaktion zwischen Explorator und Gewährsperson auf die Perzeption, die für Gessinger/Butterworth (2015: $259 \mathrm{u}$. 262) eine der „Dimensionen von „Salienz“ ist, wird in Hettler 2015 thematisiert.
} 
ausgeprägtes Sprachbewusstsein verfügen“ (Keuch/Wirrer 2014: 95), trotz bspw. unterschiedlicher Vorraussetzungen im Rahmen der Sprachsozialisation, ähnliche Salienztestergebisse aufweisen und mehr als die Hälfte der im Perzeptionstest befindlichen Varianten hören (cf. Keuch/Wirrer 2014: 95).

In Hinblick darauf, dass das Bewusstsein das Handeln mitsteuert, wird zudem häufig angenommen, dass empirisch fassbares Sprachwissen auch den Sprachgebrauch steuert (cf. Häcki Buhofer 2002: 22). Häcki Buhofer konstatiert in diesem Kontext jedoch, dass subjektives Sprachbewusstsein und objektiver Sprachgebrauch divergieren können: „Was die Sprecherinnen und Sprecher über ihren Sprachgebrauch, wissen', stimmt notwendigerweise nicht mit dem überein, was sie ,tun', wenn sie sprechen“ (Häcki Buhofer 2002: 23). So zeigen Teilergebnisse des Baseler Stadtsprachenprojektes, dass wenig Zusammenhang zwischen bewussten Sprachwissensbeständen und der Verwendung phonologischer und morphologischer Varianten besteht (cf. Häcki Buhofer 2002: 26). Auch Pratt/Grieve halten fest, dass sprachliches Wissen (metalinguistic awareness) und Sprachgebrauch nicht korrelieren müssen: "The development of awareness of language [...], does not necessarily entail its application to monitoring the use of language in all contexts" (Pratt/Grieve 1984: 9). Untersuchungen wie die von Labov zeigen hingegen, dass Sprecher bewusst besonders auffällige Merkmale ihrer Sprache kontrolliert verwenden bzw. vermeiden können, wenn es sozio-situativ angemessen erscheint (Labov 1966). Zudem kann die Verwendung bestimmter regionaler Merkmale bewusst erfolgen, um sich als Sprecher eines lokalen Dialektes bzw. einer bestimmten regionalen Varietät auszuweisen. Johnstone bezeichnet dies als „stancetaking“ (Johnstone 2007: 50) und weist darauf hin, dass Gewährspersonen in einem objektiv linguistischen Sinne keineswegs kompetente Dialektsprecher sein müssen, sondern zumeist die Realisierung weniger Merkmale ausreicht, sich als solche darzustellen (cf. Johnstone 2007: 50 u. 66). Hiermit wird auch auf die Triggerfunktion (salienter) sprachlicher Phänomene in Bezug auf die Aktivierung von regionalsprachlichen Konzepten verwiesen (cf. Anders 2010a: 54).

Da sprachliches Wissen bzw. eine ausgeprägte Sprachbewusstheit Auswirkungen auf die Sprachwahrnehmung und den Sprachgebrauch haben kann, sollen im Folgenden laienlinguistische Wissensbestände genauer betrachtet werden. Neben der Thematisierung von Termini wie „Sprachbewusstheit“, „Sprachbewusstsein“, „(meta)sprachliches Wissen“ und „language awareness“ ist dabei auch von Bedeutung, welche Faktoren die Heranbildung einer ausgeprägten Fähigkeit zur Reflexion und metasprachlichen Thematisierung von regionalen Phänomenen bei Sprechern begünstigen.

\section{Laienlinguistische Wissenbestände}

Entstehung und Entwicklung (meta)sprachlicher Wissensbestände sind insbesondere Thema der Spracherwerbs- und Mehrsprachigkeitsforschung, stehen aber auch im Fokus der Wahrnehmungsdialektologie, die u. a. zum Inhalt hat, ,sowohl die individuellen als auch die alltäglichen sprachbezogenen Wissensbestände in Form von individuellem Laienwissen und kollektivem Alltagswissen als laienlinguistisches Alltagswissen zu regionalen Varietäten“ (Anders 2010b: 73) zu untersuchen. Wissensbestände über Sprache werden in der Forschung häufig im Kontext unterschiedlicher Termini wie „Sprachwissen“, „metasprachliches Wissen“, „Sprachbewusstsein“ oder „Sprachbewusstheit“ behandelt, wobei insbesondere die 
Bezeichnungen „Sprachbewusstsein“ und „Sprachbewusstheit“ zum Teil synonym und zum Teil unterschiedlich verwendet werden (cf. Hinneberg 2005: 57). ${ }^{8}$ Häufig wird „Sprachbewusstsein“ als der übergeordnete Begriff verstanden, was bedeutet, dass „Sprachbewusstsein ohne Sprachbewusstheit funktionieren kann, Sprachbewusstheit jedoch Sprachbewusstsein voraussetzt“ (Hinneberg 2005: 61). Eine weitere, gängige Definition beschreibt „Sprachbewusstheit als konkrete metasprachliche Handlung und Sprachbewusstsein als das sprachliches Wissen, das diesen Handlungen zu Grunde liegt“ (Hug 2007: 10). Insbesondere in Studien, die sich mit dem Einfluss von Wissensbeständen auf den Zweitspracherwerb beschäftigen, wird zudem häufig der Begriff der ,language awareness" herangezogen, der seine Ursprünge in der englischen Muttersprachendidaktik hat (cf. Fehling 2010: 44) und in der Regel mit Sprachbewusstheit (cf. Wolff 1993: 511) manchmal aber auch bspw. mit Sprachbetrachtung, Sprachwissen oder Sprachbewusstsein (cf. Green/Hecht 1993: 125) übersetzt wird. Edmondson/House (1997: 4) weisen auf die Unschärfe des Begriffs hin, wenn sie ausführen, dass „language awareness“ sowohl mit Sprachwissen und einer expliziten mentalen Leistung, jedoch auch mit einem allgemeinen mentalen oder kognitiven Besitz verbunden werden kann:

Auf der einen Seite wird Language Awareness als sprachliches Wissen verstanden. Mit „Wissen“ kann explizites oder implizites Wissen gemeint sein. [...] Auf der anderen Seite wird unter Language Awareness eher eine Art kognitive Ausstattung verstanden, durch die die Besitzerin der Language Awareness eine besondere Art von rezeptiver Sensibilität von Sprache hat, vor allem durch besondere intuitive und/oder analytische Aufnahmefähigkeiten.

(Edmondson/House 1997: 4)

Wichtig für die vorliegende Studie ist der im obigen Zitat gegebene Hinweis auf die Bezeichnungen „explizites“ und „implizites Wissen“, die mit „bewusstem und unbewusstem Wissen“ (Gnutzmann 2012: 40) gleichgesetzt werden können, wobei bewusst bzw. unbewusst zwei Pole einer Skala mit „unterschiedlichen Graden von Sprach(en)bewusstheit“ bilden (Gnutzmann 2012: 40). ${ }^{9}$ Häufig werden implizites und explizites Wissen zudem mit prozeduralem und deklarativem Wissen in Verbindung gebracht, wobei es sich beim prozeduralen Wissen i. d. R. um automatisiertes Wissen handelt, das unbewusst ist, während sich das deklarative Wissen auf kommunizierbare Wissensbestände richtet (cf. Gnutzmann 2012: 40f.).

An dieser Stelle kann keine weiterführende, explizite Diskussion der unterschiedlichen Begrifflichkeiten erfolgen, festzuhalten ist jedoch für die folgenden Ausführungen, dass unter „Sprachbewusstheit“" sowohl implizite als auch explizite Wissensbestände, die sich auf Sprache beziehen, verstanden werden, und unter „metasprachlichem Wissen“ explizite, deklarative sprachbezogene Wissensbestände, die Sprechern bzw. Hörern bewusst sind und, zumindest grundsätzlich, kommuniziert werden können. Diese Verbalisierungen sind bei der Analyse von Sprachwissensbeständen direkt zugänglich, ${ }^{10}$ während die impliziten

\footnotetext{
${ }^{8}$ Cf. zu einer Übersicht über verschiedene Begriffsdefinitionen im Feld Sprachbewusstsein, Sprachbewusstheit etc. Hinneberg 2005: 57.

${ }^{9}$ Siehe zu unterschiedlichen Graden der Aufmerksamkeit, bzw. der Sprachbewusstheit (language awareness) auch Schmidt 1994.

${ }^{10}$ Diese Thematisierung läuft vorrangig verbal ab, (nonverbale) Reaktionen wie bspw. Lachen oder Stirnrunzeln können aber auch Aufschluss über die Bekanntheit, Bewertung und Einordnung von Varianten geben.
} 
Wissensbestände nicht beobachtbar sind und man über sie bestenfalls Vermutungen anstellen kann.

Die Fähigkeit, metasprachliches Wissen $\mathrm{zu}$ thematisieren, ist individuell unterschiedlich ausgeprägt. So weist DeKeyser darauf hin, dass die Fähigkeit, explizites Wissen zu verbalisieren, von kognitiven und metsprachlichen Fähigkeiten des betreffenden Sprechers abhängig ist und sich in Explizitheit und Vollständigkeit unterscheiden kann (cf. DeKeyser 2009: 121f.). Preston hält fest, dass, im Rahmen der Bewusstheit (awareness) sprachlicher Merkmale und der Thematisierung dieser, sich Sprecher darin unterscheiden, welche Aufmerksamkeit sie sprachlichen Phänomenen schenken und in welchem Maße sie sich mit ihnen beschäftigen oder sie z. B. metasprachlich thematisieren (availability). Weiterhin schwankt ihre Fähigkeit, Sprache $\mathrm{zu}$ charakterisieren (accuracy), die Beschreibung sprachlicher Merkmale divergiert in Genauigkeit und Differenziertheit (detail) und die Sprecher sind in unterschiedlichem Maße in der Lage, sprachliche Merkmale wiederzugeben bzw. zu imitieren (control) (cf. Preston 2002: 50f.). Auch im Rahmen der vorliegenden Studie ist davon auszugehen, dass nicht alle Gewährspersonen im selben Ausmaß in der Lage sind, sprachliche Abweichungen, die sie im Salienztest wahrnehmen, explizit zu benennen oder im Rahmen der qualitativen Interviews Auskunft über Merkmale der jeweiligen Stadtsprache bzw. ihre eigenen Sprache zu geben, da sie z. B. ein unterschiedliches metasprachliches Wissen aufweisen oder in der Beschreibung von sprachlichen Phänomenen unterschiedlich geübt sind.

Mögliche Faktoren, die Sprachbewusstheit im Rahmen des Spracherwerbs fördern, sind für Peter (2011: 27) „die allgemeine kognitive Entwicklung, der Schriftspracherwerb oder sprachspielerische Aktivitäten“ (Peter 2011: 27). Speziell in Bezug auf die schulische Vermittlung von (meta)sprachlichem Wissen konstatiert Häcki Buhofer, dass die Qualifikation zur Thematisierung von metasprachlichem Wissen häufig eher im Fremdsprachenunterricht gelehrt wird, im Erstsprachenunterricht dagegen ein Desiderat in Bezug auf die Vermittlung sprachwissensbezogener Inhalte vorliegt (cf. Häcki Buhofer 2002: 19). Auch Keuch/Wirrer nehmen an, dass bei dem Erwerb von Wissen über Sprache die Schule sowie populäre oder wissenschaftliche Publikationen, die sich mit Sprache beschäftigen, nur eine marginale Rolle spielen (cf. Keuch/Wirrer 2014: 69). Vielmehr ist die wichtigste Quelle für den Erwerb von Sprachwissen „die eigene Erfahrung im aktiven oder auch passiven Umgang mit Sprache“ so z. B. bei kommunikativen Erfolgen und Misserfolgen oder bei der Erfahrung von Sprachverschiedenheit (Keuch/Wirrer 2014: 68). Weiterhin von Bedeutung ist „die vermittelte sprachliche Erfahrung anderer“, insbesondere von Personen aus dem engeren Familien- bzw. Bekanntenkreis (Keuch/Wirrer 2014: 68). Auch Häcki Buhofer nimmt an, dass ein vielfältiger Sprachkontakt das Sprachbewusstsein fördert und eine positive Auswirkung auf den weiteren Spracherwerb hat (cf. Häcki Buhofer 2002: 20). Scherfer bemerkt zudem, dass der Grad, in dem Sprecher sprachliche Wissensbestände abrufen können, davon abhängig ist, in welchem Maße sie darin geübt sind, über Sprache zu reflektieren oder sie verbal zu thematisieren (cf. Scherfer 1983: 25). Die Studie von Ivo/Neuland zur Akzeptabilität grammatischer Normen thematisiert in diesem Zusammenhang den Einfluss des Parameters Beruf: So zeigt sich u. a., dass jene Gewährspersonen, die in schriftnahen Berufen arbeiten, am ehesten Normverstöße bemerken und diese auch besser als andere Befragte metasprachlich artikulieren können (cf. 
Ivo/Neuland 1991: 450-454). Auch im Rahmen der vorliegenden Studie ist zu untersuchen, ob die Ausübung bestimmte Berufe (z. B. Lehrer) mit einem erhöhten Sprachwissen und Normbewusstsein der jeweiligen Sprecher einhergeht.

In Bezug auf sprachbezogene Äußerungen und den Rückgriff, den diese auf das metasprachliche Wissen von Sprechern ermöglichen, ist immer auch zu hinterfragen, in welchen Situationen des Alltagslebens Menschen einen Anlass darin sehen, sprachliche Phänomene zu reflektieren und über sie zu sprechen, da „Sprachreflexion eng mit konkreten Problemstellungen und Bedürfnissen verknüpft ist" (Lehr 2002: 188). So trennt Wehr zwei Arten „metasprachlichen Verhaltens“ - zum einen das „spontane“ das auf persönlichem Interesse basiert und selbstgesteuert ist bzw. durch bestimmte Situationen ausgelöst wird und das „evozierte“, dass durch Außenstehende vermittelt wird und fremdinduziert ist, so z. B. in der Schule und durch Testsituationen (cf. Wehr 2001: 89). ${ }^{11}$

Allgemein bieten sowohl der im Rahmen dieser Studie durchgeführte Salienztest (siehe Kapitel 4) als auch das sprachbiografische Interview die Möglichkeit, durch die Elizitierung metasprachlicher Äußerungen Aufschlüsse über Bewertungs- und Wissensstrukturen (wie z. B. der regionalen Verortung) verschiedener Varianten $\mathrm{zu}$ erhalten, die sonst der linguistischen Analyse nicht zugänglich wären. Erkenntnisse über den Zusammenhang von Sprachperzeption/metasprachlichem Wissen und dem Sprachgebrauch lassen sich dagegen nur durch den Einbezug objektsprachlicher Daten erhalten. Im Folgenden sollen die objektiven und auch die subjektiven Daten, die als Grundlage zur Bildung von individuellen Sprecher-/Hörerprofilen und, auf diesen aufbauend, Sprecher-/Hörertypen dienen, genauer beschrieben werden.

\section{$4 \quad$ Methodischer Zugriff}

Fokus des vorliegenden Textes sind Teilergebnisse einer vorrangig qualitativ ausgelegten Studie, in deren Rahmen in Bremen und Hamburg insgesamt zwanzig Personen (vierzehn Männer und sechs Frauen) befragt wurden, von denen jeweils fünf Personen über fünfundsechzig sowie fünf Personen unter fünfunddreißig sind. Bei den Gewährspersonen handelt es sich um gebürtige Bremer und Hamburger bzw. um Personen, die seit ihrer Kindheit in Bremen oder Hamburg leben. ${ }^{12}$

Um salienzbedingende Parameter und das Zusammenspiel hörer- bzw. sprecherindividueller Faktoren zu untersuchen, wurde eine Kombination aus verschiedenen Methoden gewählt: Um den Sprachgebrauch der Befragten für eine spätere Variablenanalyse in verschiedenen

\footnotetext{
11 Auch Gombert nimmt an, dass die expliziteste Form der metasprachlichen Fähigkeit nur durch besondere Bedingungen und Anforderungen elizitiert werden kann. So kann die dritte Phase seines Metasprachmodells, die Aneignung von metasprachlicher Bewusstheit, nur dann durchlaufen werden, wenn Sprecher durch bestimmte Kommunikationssituationen, die im Alltagsleben nicht immer vorhanden sind, zur Beschäftigung mit Sprache angeregt werden. Die dritte Phase mündet in der vierten, finalen Phase, die einen Zustand bewusster Aufmerksamkeit darstellt, der auf die Sprache selbst gerichtet ist und der jederzeit hergestellt werden kann, falls es die Situation, bzw. eine bestimmte Aufgabe, erfordert (cf. Gombert 1992: 187-191).

12 Ein Ausnahmefall ist eine Bremer Gewährsperson, bei der sich im Rahmen des Interviews herausstellte, dass sie in der Nähe von Uelzen und Verden aufgewachsen ist. Da sich der Sprecher jedoch laut Eigenaussage als Bremer fühlt, die Mutter zudem aus Bremen stammt und die Gewährsperson seit 1969 auch in Bremen lebt, scheint es angemessen, ihre Daten mit in das Korpus aufzunehmen.
}

ISSN 1615-3014 
Formalitätsgraden betrachten zu können, wurden die Settings Freundesgespräch, Interview sowie Vorlesetext ausgewählt. ${ }^{13}$ Das Freundesgespräch wurde nicht für jede der zwanzig Gewährspersonen einzeln organisiert, sondern gruppenweise, so dass je fünf befreundete Gewährspersonen bei einem etwa zweistündigen gemeinsamen Treffen ohne die Anwesenheit des Explorators aufgenommen wurden. ${ }^{14}$ Für die Type-Token-Analysen wurden Ausschnitte des Familiengesprächs sowie des Interviews (2500 Wörter, soweit vorhanden) und der gesamte Vorlesetext mithilfe des Partitur-Editors EXMARaLDA transkribiert ${ }^{15}$ und in Hinblick auf alle phonetisch-phonologischen Merkmale und ausgewählte morphosyntaktische Varianten, die auch im späteren Perzeptionstest vorkommen, untersucht. Die Auswertung der annotierten Transkripte fand mittels des EXMARaLDA-Suchwerkzeugs EXAKT und der Überführung der Ergebnisse in Exceltabellen statt. Für die Untersuchung von Sprachwissens- und Spracheinstellungsbeständen der zwanzig Gewährspersonen wurden zudem die Interviewaufnahmen transkribiert. Einige Zeit nach dem Freundesgespräch wurden Einzelinterviews mit allen Gewährspersonen geführt. Es handelte sich um leitfadengestützte Interviews, die vorrangig Aufschluss über das metasprachliche Wissen der Probanden geben sollen, um diese Belege mit den Ergebnissen des Salienztests und objektsprachlichen Daten in Verbindung bringen zu können. Unter anderem wurde danach gefragt, ob es eine typische Sprechweise der Hamburger bzw. Bremer geben würde und wie diese bezeichnet wird, um auf dieser Basis das Wissen über typische Merkmale der eigenen und fremden Stadtsprache (,Was zeichnet diese Sprache aus?") und die diastratische und diaphasische Verortung der Varietät (Bsp. „Wo kann man diese Sprache hören?“, „Wer spricht diese Sprache?“) abzufragen. Direkt auf die Spracheinstellung und das Normverständnis der Gewährspersonen waren Fragen zu der Bewertung des Hamburgischen bzw. Bremischen gerichtet. ${ }^{16}$ Zudem sollten die Befragten auch ihre eigenen Sprechweise und Charakteristika dieser angeben, um u. a. betrachten zu können, ob sich die Selbstwahrnehmung der Sprecher in Bezug auf ihr Sprachverhalten im Rahmen der Type-Token-Analysen bestätigt. Am Ende des Interviews wurden zudem noch Fragen zu Bremen und Hamburg gestellt, die vorrangig dazu dienten, einen Einblick in die Ortsloyalität der Befragten zu erhalten. ${ }^{17}$ Zuletzt wurde ein Salienztest durchgeführt, in dem die Auffälligkeit, Bewertung und Verortung regionaler sprachlicher Phänomene untersucht wurde. Der Test besteht aus 34 Sätzen, von denen 33 Sätze, in einem ansonsten standardsprachlichen Kontext, jeweils eine standardabweichende Variante aufweisen. ${ }^{18}$ Bei den Merkmalen handelt es sich um phonetisch-phonologische, morpho-syntaktische

\footnotetext{
13 Siehe zu den Settings auch den Untersuchungsaufbau des SiN-Projektes (cf. Elmentaler et al. 2006: 167-169).

14 Alle Treffen fanden in einem privaten Rahmen statt: So trafen sich zwei Gruppen zum Abend- bzw. Pizzaessen, eine Gruppe zum Kaffeetrinken und eine Gruppe zum Frühstücken.

15 www.exmaralda.org. Aufgrund der Datenmenge wurde für die Transkription der Sprachaufnahmen eine orthografische, lautnahe Transkription gewählt. Zur Kennzeichnung der untersuchten Variablen bzw. Varianten wurden zum Teil die Transkriptionsregeln des Hamburgisch-Projektes (cf. Bieberstedt/Ruge/Schröder 2011 und 2016) befolgt sowie, von diesen abweichend, nach eigenen Konventionen verfahren, so z. B. in der Verschriftlichung von alveolarem [s] nach Plosiv mit „s-t“ oder von velarem /a:/ durch „oa“ (Joahren).

16 Die Bewertungsfragen sowie die Fragen zur situativen und funktionalen Verortung wurden zu allen genannten Varietäten wie z. B. Plattdeutsch oder Missingsch gestellt. Spracheinstellungen zum Varietätenspektrum in Bremen und Hamburg werden explizit in Hetter 2016 behandelt.

17 Zum Begriff Ortloyalität siehe u. a. Ziegler 1996: 241.

18 Das Vorgehen wurde gewählt, da es eine gute Kontrolle des Einzelmerkmals ermöglicht (vgl. auch Elmentaler/Gessinger/Wirrer 2010). Es können somit jedoch keine Aussagen darüber gemacht werden, inwieweit bspw.
}

ISSN 1615-3014 
und lexikalische Phänomene der Bremer und Hamburger Stadtsprache. Die meisten Phänomene sind nicht ausschließlich entweder Hamburg oder Bremen zuzuordnen, sondern betreffen in unterschiedlicher Ausprägung die regional gefärbte Sprache beider Städte oder sind weitverbreitete Merkmale der norddeutschen Umgangssprache, die auch überregional auftreten können. Die folgende Tabelle zeigt alle untersuchten Varianten, wobei in Kapitel 5 nur eine Auswahl der Variantene behandelt werden wird. ${ }^{19}$

\begin{tabular}{|c|c|c|c|}
\hline Phänomen & Salienztestvariante & Phänomen & Salienztestvariante \\
\hline Spirantisierung -urg & Hambuich & $\begin{array}{l}\text { Plosivische Real. } \\
\text {-ig }\end{array}$ & günstik \\
\hline alveolares [s] & S-törtebeker & Vokalisierung /r/ & Stöache \\
\hline Vokalkürzung /i:/ & widder & $/ \mathrm{au} / \mathrm{als}[\mathrm{ou}]$ & ouf \\
\hline Diphthongierung/o:/ & ouben & $/ \mathrm{t} /$-Elision & nich \\
\hline Assimilation /nd/ & Wunner & Lenisierung / $\mathrm{k} /$ & Wegger \\
\hline Spirantisierung /g/ & gesacht, Wech & $/ \mathrm{j} / \mathrm{als}[3] /[\mathrm{d} 3]$ & Djugend \\
\hline$/ \varepsilon: /$ als [e:] & Meedchen & Lenisierung $/ \mathrm{p} /$ & Pabbe \\
\hline Diphthongierung /e:/ & Nordsäi & $\begin{array}{l}\text { Diphthongierung } \\
\text { /ø:/ }\end{array}$ & schoin \\
\hline Rundung /I/ zu [Y] & schwümmen & wie statt als & besser wie er \\
\hline /I/ als $[\varnothing] /[œ]$ & Köhrche & Trennung AdvPro & $d a \ldots \operatorname{hin}^{20}$ \\
\hline Senkung /I/ zu $[\varepsilon]$ & Kerschen & Trennung ProAdv & $d a \ldots$ von \\
\hline $\begin{array}{l}\text { Ausfall Affrikaten- } \\
\text { komponente }\end{array}$ & Ferde & nach statt $z u$ & nach Müllers gehen \\
\hline Lenisierung $/ \mathrm{t} /$ & Reddungsfahrzeuge & Plural mit -en & Stücken \\
\hline Vokalkürzung /a:/ & Grass & Akk. statt Dat. & wie gut es einen geht \\
\hline$/ \mathrm{st} / \mathrm{als}\left[\int \mathrm{t}\right]$ & Illuschtrierte & Dat. statt Akk. & mir gelehrt \\
\hline Velarisierung/a:/ & Joahren & Lexem $u m z u$ & umzu \\
\hline
\end{tabular}

Tabelle 1: Übersicht über alle untersuchten Varianten und ihre Einbettung im Kontext des Salienztests ${ }^{21}$

\footnotetext{
die Frequenz eines Stimulus zu einer verstärkten Wahrnehmung der Variante führt, wie es sich z. B. bei Kiesewalter (2011) zeigt.

${ }^{19}$ Zur ausführlichen Thematisierung aller untersuchten Merkmale siehe Hettler 2015.

${ }^{20}$ Mit dem getrennten dahin wurde eine standardsprachliche Variante gewählt, um zu untersuchen, ob Sprecher (bewusst oder unbewusst) Spaltungskonstruktionen von Richtungsadverbien anders bewerten als die Trennung von Pronominaladverbien.

${ }^{21}$ Da die Verwendung niederdeutscher Elemente in der Umgangssprache unabhängig vom synchronen Kontakt zum Niederdeutschen ist (cf. Höder 2003: 56), werden die Varianten ausgehend vom Hochdeutschen und der Standardsprache beschrieben.
} 
Auch das Gespräch während des Salienztests wurde aufgenommen, um die zahlreichen metasprachlichen Kommentare der Gewährspersonen zu sichern. Um bei der Analyse der Ergebnisse festzuhalten, welche Gewährspersonen besonders saliente oder wenig saliente Stimuli wahrnehmen, wurden als Vergleichsfolie Salienztestergebnisse einer vorrangig quantitativ ausgerichteten Untersuchung, in der vierzig Bremer und vierzig Hamburger befragt wurden und in der sowohl Varianten ausgemacht werden konnten, die von fast allen Gewährspersonen gehört werden (bspw. das alvoelare [s]), als auch Phänomene (bspw. die Realisierung von / $\varepsilon$ :/ als [e:]), die allgemein einen sehr niedrigen Salienzgrad besitzen, herangezogen. ${ }^{22}$ Es liegen somit sowohl subjektive Daten zur Perzeption und Bewertung von Varianten sowie, durch die Analyse der Sprachaufnahmen, objektive Daten zum tatsächlichen Gebrauch der untersuchten Varianten vor, die miteinander in Bezug gesetzt werden können.

\section{$5 \quad$ Bildung von Sprecher-/Hörertypen}

Mithilfe der oben aufgeführten Daten wurden Sprecher-/Hörertypen gebildet. Als oberstes Kriterium bei der Kategorisierung wurden die Menge und die Art der perzipierten Varianten im Salienztest angesetzt, so dass sich eine Vorauswahl von drei Gruppen ergibt: Gewährspersonen, die weniger als die Hälfte der Merkmale als auffällig von der Standardsprache einordnen, Befragte, die mehr als die Hälfte, jedoch unter zwei Drittel der Merkmale perzipieren, und jene Gewährspersonen, die mehr als zwei Drittel der Stimuli als auffällig einordnen. Zudem wurde betrachtet, welche Hörer wenig saliente Merkmale wahrnehmen, welche Hörer stark saliente Merkmale nicht wahrnehmen und bei welchen die Wahrnehmung dem „Durchschnitt“ entspricht, wobei als Vergleichsgröße für ein „auffälliges“ Hörerverhalten die ermittelten Salienzgrade aus der angesprochenen quantitativen Untersuchung herangezogen wurden. ${ }^{23}$ Auch wurde miteinbezogen, welcher sprachlichen Ebene die perzipierten Merkmale zuzuschreiben sind.

Als zweites Kriterium der Einteilung in Sprecher-/Hörertypen wurden die metasprachlichen Belege aus den Interviews, wie Angaben zum Sprachwissen und zum Normverständnis der Befragten, miteinbezogen.

Das dritte Kriterium, das vorrangig zur Differenzierung von Sprechern innerhalb einer Gruppe dient, ist, ob sich die Gewährspersonen eher durch einen standardnahen Sprachgebrauch auszeichnen, oder regionale Varianten realisieren. Zur Unterteilung der Sprecher in standardnah/regional ist zu bemerken, dass hierbei nicht nur die Anzahl, sondern auch die Art der realisierten Varianten miteinbezogen und zwischen überregionalen Varianten eines norddeutschen Gebrauchsstandards und spezifischen regionalen Formen unterschieden wird: Jene Sprecher, die vorrangig Merkmale des norddeutschen Gebrauchsstandards (cf. Berend 2005) wie die Realisierung von / $\varepsilon$ :/ als [e:] sowie überregionale Varianten wie die /g/Spirantisierung und die /t/-Elision realisieren, werden $\mathrm{zu}$ den standardnahen Sprechern

\footnotetext{
${ }^{22} \mathrm{Zu}$ den Ergebnissen dieser Untersuchung und zur differenzierten Diskussion der Einzelmerkmalssalienz von Varianten und dem Vergleich mit anderen Studien siehe Hettler 2014 und Hettler 2015.

${ }^{23}$ Angaben wie „eigentlich“ bzw. „erwartbar“ salient oder nicht salient beziehen sich also auf die Ergebnisse dieser Studie. Zu der Spanne perzipierter Merkmale bei den 80 Gewährspersonen und einem Vergleich mit dem Sample der 20 Befragten, die hier im Fokus stehen, siehe Hettler 2015.
} 
gezählt. ${ }^{24}$ Im Gegensatz dazu stehen Sprecher, die zusätzlich noch deutlich als regional zu charakterisierende Merkmale wie bspw. die /a:/-Velarisierung, die Lenisierung von Konsonanten oder die Diphthongierung von Vokalen in ihrem Sprachgebrauch aufweisen. ${ }^{25}$

Drei Gruppen konnten ausgemacht werden: Die „Sprachbewussten“ (7 Gewährspersonen), die „Regionsverwachsenen und Sprachvarianztoleranten“ (5 Gewährspersonen) und die „Durchschnittshörer“ (6 Gewährspersonen). ${ }^{26} \mathrm{Im}$ Rahmen dieser Studie wird für diese Sprecher-Hörertypen, bzw. die jeweiligen Untertypen dieser, exemplarisch das tabellarische Perzeptions-, Wissens- und Sprachprofil einer ausgewählten Gewährsperson gezeigt, die als Prototyp für den jeweiligen Typus gelten kann. Allgemein ist $\mathrm{zu}$ vermerken, dass die einzelnen Sprecher-/Hörertypen nur eine grobe Gruppierung darstellen können, da jede Gewährsperson individuelle Wissensbestände, Sprachmuster und sprachbiografische Hintergründe besitzt. Solche individuellen Unterschiede werden innerhalb der Gruppen diskutiert.

Die Tabellen, die unter 5.1 bis 5.3 gezeigt werden, sind folgendermaßen aufgebaut: Zunächst ist neben der Sigle der Gewährsperson vermerkt, wieviele Varianten insgesamt im Salienztest gehört wurden. Unter „Perzeption“ wurde vermerkt, welche Stimuli als auffällig $(+=$ ja) kommentiert wurden, wobei die Spirantisierung /g/ zwei Werte aufweist, da sich der erste Wert auf den Stimulus gesacht und der zweite auf den Stimulus Wech bezieht. Unter „Wissen“ wurde vermerkt, ob die Variante im Rahmen des Interviews als ein Merkmal der Bremer oder Hamburger Stadtsprache bzw. der eigenen Sprache genannt wurde $(+=\mathrm{ja}) .{ }^{27}$ Ein eingeklammertes Plus (+) bedeutet, dass es unklar ist, ob sich die Gewährsperson explizit metasprachlich auf ein Merkmal bezieht oder ob sie eine Variante als standardabweichend eingeordnet hat. ${ }^{28}$ Es folgt der prozentuale Anteil standarddivergenter Varianten in Prozent (SdV prozentual) sowie die Anzahl standardsprachlicher (SV absolut) und standarddivergenter (SdV absolut) Varianten in absoluten Zahlen. ${ }^{29}$ Da im morphosyntaktischen Bereich nur der Komparativgebrauch sowie die Verwendung des Pronominaladverbs und Richtungsadverbs mittels einer Variablenanalyse untersucht wurden, sind für die restlichen Stimuli dieser Ebene keine Werte in den Spalten SdV prozentual, SV

\footnotetext{
${ }^{24}$ Auch Höder (2003: 51) ordnet viele dieser Merkmale als regiolektal etabliert ein.

${ }^{25}$ Siehe zu einer Diskussion der untersuchten Varianten in Hinblick auf ihre regionale Verbreitung auch Hettler 2015.

${ }^{26}$ Zwei der untersuchten Gewährspersonen gelten als Sonderfälle und konnten keiner Gruppe zugeordnet werden. Beide Gewährspersonen nehmen zwar 21 Stimuli wahr und könnten somit in die Gruppe der Sprachbewussten eingeordnet werden, die Art der perzipierten Varianten ist aber sehr gemischt, da unter anderem Varianten, die für fast alle Gewährspersonen (und auch für die Probanden in der Gruppe der Sprachbewussten) im Rahmen der quantitativen Analysen salient waren, nicht gehört werden. Gleichzeitig werden Phänomene wie der Ausfall der Affrikatenkomponente und die Rundung von / / / zu [Y], die in der Regel nur von sehr sprachbewussten Probanden wahrgenommen werden, gehört, so dass auch eine Einordnung in die Gruppe der Durchschnittshörer nicht sinnvoll wäre. Zu einer genaueren Diskussion der beiden Befragten siehe auch Hettler 2015.

${ }^{27}$ Bei nicht-Nennung des Merkmals wird dagegen kein ,,““ eingetragen, da keine Angaben über etwaiges vorhandenes Wissen gemacht werden können, das nicht thematisiert wird.

${ }^{28}$ So ist z. B. insbesondere bei der Einordnung des getrennten Pronominaladverbs häufig unklar, welcher Teil des Satzes bzw. der Präposition für die Gewährspersonen von der Standardsprache abweichend ist.

${ }^{29}$ Die Werte wurden hier für alle Settings zusammengefasst. Zur Realisierung regionaler Varianten nach Settings siehe Hettler 2015.
}

ISSN 1615-3014 
absolut und SdV absolut vermerkt. Im Folgenden sollen sechs ausgewählte Gewährspersonen aus den drei Gruppen genauer betrachtet werden. ${ }^{30}$

\section{$5.1 \quad$ Die Sprachbewussten}

Die Gruppe der Sprachbewussten zeichnet sich dadurch aus, dass die Gewährspersonen dieser Gruppe zwei Drittel oder mehr der Salienzteststimuli wahrnehmen und mindestens ein Merkmal als salient beurteilen, das im Rahmen der quantitativen Studien als nur mäßig oder wenig auffällig eingeordnet wurde. $\mathrm{Zu}$ den Sprachbewussten zählen, bis auf jeweils zwei Ausnahmen, nur Bremer Gewährspersonen, sowie ausschließlich jüngere Sprecher aus den Gruppen der unter Fünfunddreißigjährigen, was auch auf die Korrelation zwischen Alter und Sprachwahrnehmung hinweist. ${ }^{31}$

Im Folgenden wird zunächst das Sprecher-/Hörerprofil von GP14-HH exemplarisch betrachtet. Die Tabelle zeigt hierbei 23 ausgewählte untersuchte Varianten an, ${ }^{32}$ als Grundlage für die Einordnung des Perzeptionsverhaltens der Befragten dienen jedoch die Ergebnisse des gesamten Salienztests (33 Varianten).

\begin{tabular}{|l|c|c|c|c|c|}
\hline GP14-HH (23/33) & Perzeption & Wissen & $\begin{array}{c}\text { SdV pro- } \\
\text { zentual }\end{array}$ & $\begin{array}{c}\text { SV } \\
\text { absolut }\end{array}$ & $\begin{array}{c}\text { SdV ab- } \\
\text { solut }\end{array}$ \\
\hline Spirantisierung -urg & + & & $0,0 \%$ & 2 & 0 \\
\hline alveolares [s] & + & & $0,0 \%$ & 99 & 0 \\
\hline Diphthongierung/o:/ & & & $3,1 \%$ & 343 & 11 \\
\hline Assimilation/nd/ & + & & $6,3 \%$ & 59 & 4 \\
\hline Spirantisierung/g/ & ++ & + & $46,2 \%$ & 35 & 30 \\
\hline /ع:/ als [e:] & & & $92,1 \%$ & 3 & 35 \\
\hline Diphthongierung/e:/ & & + & $3,2 \%$ & 209 & 7 \\
\hline Rundung/I/ zu [r] & & & $6,6 \%$ & 285 & 20 \\
\hline Ausfall Affrikatenkomp. & + & & $100,0 \%$ & 0 & 3 \\
\hline Lenisierung/t/ & + & + & $31,3 \%$ & 114 & 52 \\
\hline Vokalkürzung/a:/ & + & & $25,0 \%$ & 3 & 1 \\
\hline Velarisierung/a:/ & + & + & $11,0 \%$ & 395 & 49 \\
\hline Vokalisierung/r/ & + & & $0,4 \%$ & 242 & 1 \\
\hline /t/-Elision & + & + & $92,4 \%$ & 6 & 73 \\
\hline Lenisierung/k/ & + & + & $8,0 \%$ & 46 & 4 \\
\hline Lenisierung/p/ & + & & $15,4 \%$ & 11 & 2 \\
\hline wie statt als & + & & $0,0 \%$ & 6 & 0 \\
\hline
\end{tabular}

${ }^{30}$ Zur vollständigen Behandlung aller untersuchten Varianten sowie zur ausführlichen Diskussion aller zwanzig Gewährspersonen siehe Hettler 2015.

31 So zeigt sich bei Hettler 2015, dass generell die Hörer unter sechsundvierzig eher Merkmale wahrnehmen, als die Gewährspersonen, die älter sind, wobei die aufgezeigte Hierarchie in hohem Maße für die Wahrnehmung lautlicher Merkmale, jedoch weniger für morpho-syntaktische Phänomene gilt, bei denen die Werte von der jüngsten bis zur ältesten Altersgruppe im Vergleich weniger deutlich abnehmen.

32 Die Spirantisierung von /g/ zählt hierbei doppelt, da sie in zwei Stimuli abgefragt wurde. 


\begin{tabular}{|l|c|l|c|c|c|}
\hline \multicolumn{1}{|c|}{ GP14-HH (23/33) } & & & & & \\
\hline & Perzeption & Wissen & $\begin{array}{c}\text { SdV pro- } \\
\text { zentual }\end{array}$ & $\begin{array}{c}\text { SV } \\
\text { absolut }\end{array}$ & $\begin{array}{c}\text { SdV ab- } \\
\text { solut }\end{array}$ \\
\hline Trennung ProAdv & & & $22,2 \%$ & 7 & 2 \\
\hline nach statt $z u$ & + & & & & \\
\hline Plural mit - en & + & & & & \\
\hline Akk. statt Dat. & & & & & \\
\hline Dat. statt Akk. & + & & & & \\
\hline
\end{tabular}

Tabelle 2: Sprecher-/Hörerprofil von GP14-HH (Ausschnitt)

GP14-HH ist männlich und wurde 1985 in Hamburg geboren. Er besitzt Abitur und studiert VWL. Er ist mit 23 perzipierten Merkmalen die Hamburger Gewährperson, die am meisten Stimuli als von der Standardsprache abweichend eingeordnet hat. Im Vergleich zu anderen Befragten thematisiert GP14-HH überdurchschnittlich viele Merkmale des „Hamburgerisch[en]“", so z. B. die Lenisierung von /t/ und /k/ (,Man macht aus'm Doppel-t "nen Doppel-d; ,[Dass] aus 'm k eher ' $\mathrm{n}$ g [gemacht wird]"), die Spirantisierung von /g/ ("g zum ch machen“), die Diphthongierung von /e:/: („Ein langes ä aus 'm e [machen]“) oder die / $\mathrm{t}$-Elision (,[dass man] das $\mathrm{t}$ am Ende eigentlich so gut wie gar nicht spricht"). Zum Teil werden die Phänomene anhand von Beispielen verdeutlicht, so das velarisierte /a:/ anhand des Lexems joa. Die Gewährsperson gibt an, „Hamburgerisch“ zu sprechen und auch selbst die genannten Merkmale zu verwenden. Die Spracheinschätzung von GP14-HH stimmt mit den objektiven Daten weitestgehend überein: So zeigt sich bspw. eine vergleichsweise relativ starke Lenisierungstendenz bei /t/ (31,3\%), eine schwächere bei / $\mathrm{k} /$ und velarisiertes /a:/ wird mit 49 Belegen (11\%) insgesamt häufiger als bei anderen Gewährspersonen der jüngeren Altersgruppe realisiert. ${ }^{33} \mathrm{Im}$ Salienztest nimmt der Befragte mit der Ausnahme des diphthongierten /e:/ alle Merkmale wahr, die er selbst genannt hat, selbst die eigentlich nicht saliente / $\mathrm{t}$-Elision. Auch andere wenig saliente Stimuli wie das vokalisierte $/ \mathrm{r} /$ werden wahrgenommen.

Die im Vergleich zu anderen Sprechern ausgesprochen differenzierte Selbstwahrnehmung von GP14-HH und die damit verbundene vergleichsweise hohe Menge an Merkmalen, die explizit benannt werden können, ist, wie eine Analyse des Interviews nahelegt, zu einem großen Teil extern motiviert. So wurde die Gewährperson von einem Bekannten auf die eigene Sprechweise aufmerksam gemacht, da dieser ihn nach einer gehaltenen Rede auf einer Hochzeit als „Urhamburger“ bezeichnete. Die Aufnahme der Ansprache nutze die GP, um sich mit der eigenen Sprache zu beschäftigen, die während der Rede tatsächlich ,arg hamburgerisch“ gewesen sei. Bei GP14-HH ist also der von Keuch/Wirrer thematisierte Zusammenhang von Sprachbewusstheit und Spracherfahrung besonders deutlich (siehe Kapitel 3). Wie andere Gewährspersonen, die viele Merkmale hören, scheint die Gewährsperson zudem ein allgemeines Interesse an Sprache zu haben, was sich $u$. a. in der Kenntnis und dem Erlernen mehrerer Fremdsprachen äußert. Dies deckt sich mit Häcki Buhofers Einschätzung, dass nicht nur vielfältiger Sprachkontakt, sondern auch ein

\footnotetext{
${ }^{33}$ Nicht genannte Charakteristika der eigenen Sprache, für die Belege in den objektiven Daten existieren, sind u. a. die Reduktion der Affrikatenkomponente, bei der die Gewährsperson zu $100 \%$ die standardabweichende Variante gebraucht und leichte Diphthongierungstendenzen bei /o:/.
} 
vielfältiger Spracherwerb metasprachliche Fähigkeiten fördern können (cf. Häcki Buhofer 2002: 20).

Während bei GP14-HH die ausgeprägte Fähigkeit zur Sprachreflexion- und -perzeption u. a. durch die Konfrontation mit der eigenen Sprache ausgelöst wurde, hängt diese bei anderen Gewährspersonen mit einem ausgesprochenen Interesse an der Regionalsprache oder einer starken Normbewusstheit bezüglich der Standardsprache zusammen. Dies ist z. B. bei GP20BR aus Bremen der Fall.

\begin{tabular}{|c|c|c|c|c|c|}
\hline \multirow[t]{2}{*}{ GP20-BR (28/33) } & \multirow[b]{2}{*}{ Perzeption } & \multirow[b]{2}{*}{ Wissen } & \multirow[b]{2}{*}{$\begin{array}{c}\text { SdV pro- } \\
\text { zentual }\end{array}$} & \multirow[b]{2}{*}{$\begin{array}{c}\text { SV } \\
\text { absolut }\end{array}$} & \multirow[b]{2}{*}{$\begin{array}{c}\text { SdV ab- } \\
\text { solut }\end{array}$} \\
\hline & & & & & \\
\hline Spirantisierung -urg & + & & $0,0 \%$ & 23 & 0 \\
\hline alveolares [s] & + & + & $10,0 \%$ & 117 & 13 \\
\hline Diphthongierung /o:/ & + & & $0,0 \%$ & 394 & 0 \\
\hline Assimilation /nd/ & + & & $2,7 \%$ & 72 & 2 \\
\hline Spirantisierung /g/ & $+;+$ & & $23,7 \%$ & 45 & 14 \\
\hline$/ \varepsilon: /$ als [e:] & & & $81,6 \%$ & 9 & 40 \\
\hline Diphthongierung /e:/ & + & & $0,0 \%$ & 370 & 0 \\
\hline Rundung /I/ zu [Y] & & & $1,7 \%$ & 412 & 7 \\
\hline Ausfall Affrikatenkomp. & & & $40,0 \%$ & 3 & 2 \\
\hline Lenisierung /t/ & + & & $1,0 \%$ & 201 & 2 \\
\hline Vokalkürzung /a:/ & + & & $0,0 \%$ & 3 & 0 \\
\hline Velarisierung/a:/ & + & + & $0,0 \%$ & 555 & 0 \\
\hline Vokalisierung /r/ & + & & $0,8 \%$ & 263 & 2 \\
\hline /t/-Elision & + & + & $74,2 \%$ & 16 & 46 \\
\hline Lenisierung /k/ & + & & $0,0 \%$ & 61 & 0 \\
\hline Lenisierung $/ \mathrm{p} /$ & + & & $0,0 \%$ & 7 & 0 \\
\hline wie statt als & + & + & $0,0 \%$ & 6 & 0 \\
\hline Trennung ProAdv & + & + & $14,3 \%$ & 6 & 1 \\
\hline nach statt $z u$ & + & & & & \\
\hline Plural mit -en & + & & & & \\
\hline Akk. statt Dat. & + & $(+)$ & & & \\
\hline Dat. statt Akk. & + & $(+)$ & & & \\
\hline
\end{tabular}

Tabelle 3: Sprecher-/Hörerprofil von GP20-BR (Ausschnitt)

GP20-BR ist männlich und wurde 1981 geboren. Er besitzt Abitur und pendelt zwischen Bremen und Hamburg, da er in Hamburg Theologie studiert. GP20-BR ist diejenie Gewährsperson, die am meisten Salienztestmerkmale von allen Befragten hört (28 von 33), darunter auch eigentlich wenig saliente Merkmale wie das diphthongierte /o:/, die /t/-Elision, die /r/-Vokalisierung und die Vokalkürzung von /a:/. Ungewöhnlich für die jüngere Gruppe kommentiert er auch alle morpho-syntaktischen Phänomene im Salienztest. In Bezug auf die untersuchten Varianten ist GP20-BR ein sehr standardnaher Sprecher - neben überregionalen standarddivergenten Formen wie der /g/-Spirantisierung, die allerdings einen vergleichsweise niedrigen Wert aufweist (23,7\%), gibt es nur wenige von der Standardsprache abweichende 
Belege, wie z. B. den Ausfall der Affrikatenkomponente (40\%) oder die Trennung des Pronominaladverbs damit. Die Befunde decken sich mit der Selbsteinschätzung der Gewährsperson, die zwar annimmt, „,regionale Färbungen“ zu besitzen, jedoch bei Bedarf auch „recht reines Hochdeutsch“ sprechen zu können, auf das er auch Wert legt. ${ }^{34}$ Der Befragte interessiert sich jedoch auch für das Niederdeutsche und gibt an, selber gelegentlich „Platt“ im Rahmen seiner Kirchengemeinde zu sprechen. Als lautliche Merkmale für Bremen werden das „nicht gesprochene $\mathrm{t}^{\prime \prime}$, das velare /a:/ (joaa) sowie das alveolare [s] (,das st nicht als scht sprechen") genannt. Verstärkt werden auch morpho-syntaktische Phänomene thematisiert, wie die Verwechslung von wie und als oder die Trennung von Pronominaladverbien wie damit. Die Bedeutung, die ein grammatisch korrekter Sprachgebrauch für GP20-BR hat, zeigt u. a. folgendes Zitat: „Also wenn jemand mit seiner eigenen Sprache überhaupt nich umgehen kann, dann ist das wirklich... wie so Nadelstiche.“ Dementsprechend kommentiert die Gewährsperson auch die morpho-syntaktischen Stimuli im Salienztest extrem kritisch. ${ }^{35}$

Zusammenfassend verbindet die Gruppe der Sprachbewussten ein hohes metasprachliches Wissen, dass z. T. auch durch die Erziehung oder Berufswahl geprägt zu sein scheint oder, wie bei GP14-HH, extern motiviert ist. ${ }^{36}$ Bei den meisten Gewährspersonen dieses Typs lässt sich zudem ein ausgeprägtes Interesse an der Sprache der eigenen Stadt konstatieren sowie ein bewusster Umgang mit regionalen Varianten. Diese Faktoren resultieren in einer guten Einschätzung des eigenen Sprachgebrauchs sowie einer erhöhten Perzeption regionaler Merkmale im Salienztest, in dem auch selbst verwendete Varianten wahrgenommen werden. Die zweite Sprecher-Hörer Gruppe, die im Folgenden thematisiert werden soll, zeichnet sich dagegen dadurch aus, dass sie nur wenige Merkmale im Salienztest wahrnimmt.

\subsection{Die Regionsverwachsenen und Sprachvarianztoleranten}

Die Gewährspersonen dieser Gruppe hören weniger als die Hälfte der Merkmale im Salienztest. ${ }^{37}$ Zusätzlich nehmen sie auch Varianten nicht wahr, die im Rahmen der

\footnotetext{
${ }^{34}$ Die Selbsteinschätzung der Gewährsperson in Bezug auf eine situativ unterschiedliche Sprachverwendung bestätigt sich insofern in den objektiven Daten, als die wenigen standarddivergenten Merkmale wie die /g/Spirantisierung oder der Ausfall der Affrikatenkomponente in größerem Maße im Freundesgespräch auftreten und weniger frequent im Interview. Lediglich die schwer zu steuernde Realisierung von /ع:/ als [e:] ist nicht situativ verteilt.

35 Phonetisch-phonologische Varianten werden dagegen zumeist positiv oder auch neutral bewertet.

${ }^{36}$ Der Einfluss des Berufs zeigt sich insbesondere bei anderen, hier nicht thematisierten, Gewährspersonen in der Gruppe der Sprachbewussten, die z. B. Lehrer oder Erzieher sind, wobei diese Berufe nicht in jedem Fall, wie u. a. die unter 5.2 thematisierte Gewährsperson GP7-BR zeigt, automatisch ein hohes metasprachliches Wissen forcieren.

37 Aufgrund der wenigen Merkmale, welche die Probanden im Salienztest perzipieren, könnten die Gewährspersonen dieser Gruppe auch als „nicht Sprachbewusste“ oder „Sprachunbewusste“ und somit terminologisch als direkter Gegensatz der Sprachbewussten gesehen werden. Da das metasprachliche Wissen der Befragten aber vorrangig in Bezug auf regionale Sprache untersucht wurde und die Gewährspersonen metasprachliche Wissensbestände besitzen können, die nicht verbalisiert werden (konnten), schienen solche, auch evtl. stigmatisierenden, Bezeichnungen unangebracht. Da die Gründe für das Perzeptionsverhalten der Befragten zudem unterschiedlich sind, wurde einerseits der Begriff „Regionsverwachsene“ gewählt, um zu verdeutlichen, dass die (auch eigene) regionale Umgangssprache so vertraut ist, dass sie als Standard angesehen wird und andererseits die Bezeichnung „Sprachtolerante“ gewählt, die darauf hinweist, dass bei der Perzeption und Bewertung von Sprache be-
}

ISSN 1615-3014 
quantitativen Untersuchungen als stark salient ausgemacht wurden. Im Folgenden soll das Profil von GP11-HH betrachtet werden, der als Prototyp eines „regionsverwachsenen“ Sprechers gesehen werden kann, für den die Merkmale der regionalen Umgangssprache und der eigenen Sprache so vertraut sind, dass sie als Standard eingeordnet werden.

\begin{tabular}{|c|c|c|c|c|c|}
\hline GP11-HH (12/33) & & & & & \\
\hline & Perzeption & Wissen & $\begin{array}{c}\text { SdV prozentu- } \\
\text { al }\end{array}$ & $\begin{array}{c}\text { SV } \\
\text { absolut }\end{array}$ & $\begin{array}{c}\text { SdV ab- } \\
\text { solut }\end{array}$ \\
\hline Spirantisierung -urg & + & & $12,5 \% / 12,5 \% 38$ & 6 & $1 ; 1$ \\
\hline alveolares [s] & + & + & $1,0 \%$ & 97 & 1 \\
\hline Diphthongierung /o:/ & & & $2,3 \%$ & 303 & 7 \\
\hline Assimilation /nd/ & & & $2,7 \%$ & 36 & 1 \\
\hline Spirantisierung /g/ & $0 ;+$ & & $43,2 \%$ & 25 & 19 \\
\hline$/ \varepsilon: /$ als [e:] & & & $92,6 \%$ & 2 & 25 \\
\hline Diphthongierung /e:/ & + & & $0,0 \%$ & 167 & 0 \\
\hline Rundung /I/ zu [Y] & & & $6,5 \%$ & 245 & 17 \\
\hline $\begin{array}{l}\text { Ausfall Affrikaten- } \\
\text { komp. }\end{array}$ & & & $100,0 \%$ & 0 & 5 \\
\hline Lenisierung / $/ \mathrm{t}$ & & & $49,2 \%$ & 66 & 64 \\
\hline Vokalkürzung /a:/ & & & $60,0 \%$ & 2 & 3 \\
\hline Velarisierung /a:/ & + & & $5,2 \%$ & 402 & 22 \\
\hline Vokalisierung /r/ & & & $1,3 \%$ & 155 & 2 \\
\hline /t/-Elision & & & $98,7 \%$ & 1 & 78 \\
\hline Lenisierung / $/ \mathrm{k}$ & & & $31,7 \%$ & 28 & 13 \\
\hline Lenisierung /p/ & & & $20,0 \%$ & 8 & 2 \\
\hline wie statt als & + & & $0,0 \%$ & 4 & 0 \\
\hline Trennung ProAdv & & & $0,0 \%$ & 10 & 0 \\
\hline nach statt $z u$ & + & & & & \\
\hline Plural mit -en & + & & & & \\
\hline Akk. statt Dat. & & & & & \\
\hline Dat. statt Akk. & & & & & \\
\hline
\end{tabular}

Tabelle 4: Sprecher-/Hörerprofil von GP11-HH (Ausschnitt)

GP11-HH ist männlich und wurde 1985 in Hamburg geboren. Er besitzt Abitur, hat eine kaufmännische Ausbildung absolviert und ist im Groß- und Außenhandel tätig.

GP11-HH hört mit 12 perzipierten Merkmalen am zweitwenigsten Stimuli im Korpus, und ist einer der Sprecher, die den höchsten Anteil an standarddivergenten Formen aufweisen. Unter

stimmte Ebenen nicht fokussiert werden, so z. B. die lautliche. Die Wahl von zwei Begriffen legt zwar nahe, auch zwei Gruppen zu wählen, da beide (Unter)Gruppen jedoch ein ähnliches Perzeptionsverhalten an den Tag legen und sich zudem auch bei den vornehmlich Regionsverwachsenen individuell eine gewisse „Sprachtoleranz" feststellen lässt (siehe die Ausführungen zu GP11-HH) werden die betreffenden Sprecher als ein Typ mit zwei internen Ausprägungen behandelt.

38 Hier liegen sowohl Werte für die Realisierung von Hambuich als auch von Hamburch (ohne Vokalisierung von $/ \mathrm{r} /$ ) vor. 
anderem zeichnet sich sein Sprachprofil durch eine starke Lenisierungstendenz aus: So liegen die prozentualen Werte für die standarddivergente Lenisierung von /t/ bei 49,2\% und die Werte für die Lenisierung von $/ \mathrm{k} /$ bei immerhin 31,7\%, was im Vergleich zu den anderen Gewährspersonen im Korpus hoch ist. Der Anteil an spirantisierten /g/-Formen ist mit 43,2 \% der zweithöchste in der jüngeren Gruppe. Auch finden sich u. a. Belege für velarisiertes /a:/ oder Diphthongierungen. Die Gewährsperson ordnet im Salienztest zwar die Stimuli Joahren und Wech als standardabweichend ein, hört jedoch keine der, eigentlich salienten, Lenisierungen - hier ist eine Korrelation mit dem eigenen Sprachgebrauch zu konstatieren. Der beobachtete Anteil standardabweichender Varianten stimmt nicht mit der Selbsteinschätzung von GP11-HH überein, der sich keiner regionalen Eigenheiten der eigenen Sprache bewusst ist und annimmt, dass er, (wie auch in Hamburg allgemein) Hochdeutsch, „wie es geschrieben wird“, spricht. In Bezug auf regionale Merkmale ist GP11-HH nur das „S-tolpern über den s-pitzen s-tein“ bekannt, das er aber der Sprache der Hafenarbeiter vor etwa 30 Jahren zuordnet. Im Interview wird deutlich, dass sich der Befragte wenig für die Themen „Sprache“ oder „Regionalsprache“ interessiert und auch die Sprache anderer für ihn nicht wichtig ist („Nö, interessier ich mich absolut nich für"). Auch das Kriterium der Korrektheit von Sprachäußerungen ist nicht von großer Bedeutung, da grammatikalische Unsauberkeiten der Normalfall wären. So kommentiert GP11-HH die Frage, wie er „schlechtes Deutsch“ bewerte: „Das is ja, würd ich sagen, schon an der Tagesordnung. Eigentlich von daher: Links rein, rechts raus...". Bei GP11-HH ist festzuhalten, dass selbst gebrauchte regionale Varianten der Gewährsperson nicht bewusst (da vetraut) sind und Sprache, auch die regionale, nur selten Gegenstand von Reflexion ist.

In der Gruppe, die wenig Varianten im Salienztest hören, finden sich jedoch auch standardnahe Sprecher, deren geringe Perzeption u. a. dadurch beeinflusst wird, dass trotz des Interesses an der regionalen Sprache nur wenig Wissen über lautliche Varianten vorherrscht. Stattdessen werden Aspekte wie die Wortwahl oder die morpho-syntaktische Korrektheit von Sätzen im Test fokussiert und auch im Interview thematisiert. Ein Beispiel für diese „Sprachvarianztoleranten“ ist GP7-BR aus Bremen.

\begin{tabular}{|l|c|c|c|c|c|}
\hline GP7-BR (16/33) & Perzeption & Wissen & $\begin{array}{c}\text { SdV pro- } \\
\text { zentual }\end{array}$ & $\begin{array}{c}\text { SV } \\
\text { absolut }\end{array}$ & $\begin{array}{c}\text { SdV ab- } \\
\text { solut }\end{array}$ \\
\hline Spirantisierung -urg & + & & $75,0 \%$ & 1 & 3 \\
\hline alveolares [s] & + & + & $0,7 \%$ & 148 & 1 \\
\hline Diphthongierung /o:/ & & & $0,0 \%$ & 333 & 0 \\
\hline Assimilation/nd/ & & & $1,1 \%$ & 90 & 1 \\
\hline Spirantisierung/g/ & & & $22,9 \%$ & 27 & 8 \\
\hline /ع:/ als [e:] & & & $78,6 \%$ & 9 & 33 \\
\hline Diphthongierung/e:/ & & & $0,4 \%$ & 227 & 1 \\
\hline Rundung/I/ zu [y] & & + & $0,7 \%$ & 288 & 2 \\
\hline Ausfall Affrikatenkomp. & & & $42,9 \%$ & 4 & 3 \\
\hline Lenisierung/t/ & + & & $0,6 \%$ & 174 & 1 \\
\hline Vokalkürzung/a:/ & & & $33,3 \%$ & 2 & 1 \\
\hline Velarisierung/a:/ & & + & $0,6 \%$ & 341 & 2 \\
\hline
\end{tabular}




\begin{tabular}{|l|c|c|c|c|c|}
\hline GP7-BR (16/33) & Perzeption & Wissen & $\begin{array}{c}\text { SdV pro- } \\
\text { zentual }\end{array}$ & $\begin{array}{c}\text { SV } \\
\text { absolut }\end{array}$ & $\begin{array}{c}\text { SdV ab- } \\
\text { solut }\end{array}$ \\
\hline Vokalisierung/r/ & & & $0,0 \%$ & 188 & 0 \\
\hline /t/-Elision & & & $64,3 \%$ & 20 & 36 \\
\hline Lenisierung /k/ & + & & $0,0 \%$ & 41 & 0 \\
\hline Lenisierung /p/ & + & & $0,0 \%$ & 5 & 0 \\
\hline wie statt als & + & & $0,0 \%$ & 3 & 0 \\
\hline Trennung ProAdv & + & & $0,0 \%$ & 7 & 0 \\
\hline nach statt $z$ u & + & & & & \\
\hline Plural mit -en & + & & & & \\
\hline Akk. statt Dat. & & $(+)$ & & & \\
\hline Dat. statt Akk. & + & $(+)$ & & & \\
\hline
\end{tabular}

Tabelle 5: Sprecher-Hörerprofil von GP7-BR (Ausschnitt)

GP7-BR ist weiblich und wurde 1939 geboren. Sie besitzt Abitur, hat Deutsch und Geschichte studiert und als Fremdsprachenassistentin und Lehrerin gearbeitet. GP7-BR gehört, wie GP20-BR aus der Gruppe der Sprachbewussten, zu den Gewährspersonen, die wenige standardabweichende Varianten verwenden. So liegt bspw. der Wert für spirantisiertes /g/ mit nur $25 \%$ Prozent deutlich niedriger als die anderer Befragter. ${ }^{39}$ Anders als bei GP20-BR führt die Standardnähe jedoch nicht dazu, dass regionale Merkmale vermehrt als fremd oder auffällig eingeordnet werden, da GP7-BR nur 16 der 33 Stimuli als vom Standard abweichend bewertet. Während sie fast alle morpho-syntaktischen Merkmale kommentiert, fallen ihr eigentlich saliente Stimuli wie Nordsäi und Joahren nicht auf. GP7-BR kann mehr regionale Sprachmerkmale benennen als GP11-HH, so „das spitze s“, das sie auch im Salienztest wahrnimmt, sowie die Rundung von /i/ (Bsp. „Schürm“) und, als Merkmal des Hamburgischen, die Aussprache von $a$ - im Test werden die beiden letzten Varianten jedoch nicht als auffällig bewertet. Anders als bei GP11-HH kann bei der ehemaligen Deutschlehrerin kein mangelndes allgemeines Interesse an Sprache zur Erklärung der Salienztestergebnisse herangezogen werden - so gibt sie im Interview an, dass in ihrem Elternhaus viel Wert auf Grammatik und Wortschatz gelegt wurde. Auch für regionale Sprache interessiert sie sich und verfolgt z. B. die Kolumne im Weserkurier „Sprechen Sie Bremisch?".40 Allerdings scheint der Perzeptionsfokus der Gewährsperson mehr auf grammatikalischen, stilistischen und prosodischen Sprachmerkmalen zu liegen: So gibt GP7BR an, dass es „schwierig“ wäre, Charakteristika der eigenen Sprache zu nennen. Mit (lautlichen) Merkmalen hätte sie sich nie sonderlich „beschäfticht“ und bei anderen würde sie eher auf Grammatik und Wortschatz achten. Auch in ihrer Tätigkeit als Lehrerin war für sie

\footnotetext{
39 In Bezug auf den Vorlesetext ist zu vermerken, dass GP7-BR mit nur 9 standarddivergenten Belegen die Gewährsperson mit der geringsten Merkmalsdichte für dieses Setting ist. Weitere standarddivergenten Merkmale, die sich bei GP7-BR finden, sind u. a. der Ausfall der Affrikatenkomponente (42,9\%), der Ausfall von /t/ (65\%), die Kürzung von /a:/ die allerdings ausschließlich lexemgebunden stattfindet (Glas (Wein) aber nicht im Fensterglas), sowie die lexemgebundene Realisierung der Form „Hambuich“.

40 Anders als Keuch/Wirrer konstatieren, werden also, zumindest im Rahmen der vorliegenden Studie, populärwissenschaftliche Publikationen zum Thema Sprache von Sprechern durchaus wahrgenommen. Siehe Kapitel 3.
}

ISSN 1615-3014 
wichtig, „dass die Kinder richtich Deutsch sprechen und gutes Deutsch und einen guten

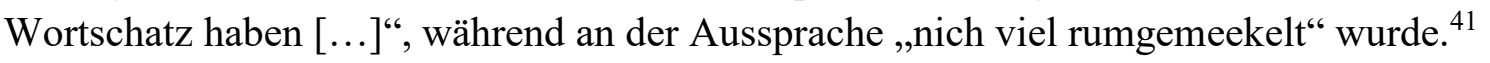

Allgemein ist es schwieriger, Gründe für die niedrige Anzahl an perzipierten Belegen in der Gruppe der Regionsverwachsenen und Sprachvarianztoleranten zu eruieren, als die Hintergründe für die ausgeprägte Wahrnehmung der Sprachbewussten zu erklären, da bei nicht perzipierten Varianten im Einzelfall natürlich auch Faktoren, wie z. B. eine geringe Aufmerksamkeit der Befragten während des Tests eine Rolle spielen können. Das Interesse, das GP7-BR an regionaler Sprache hat und das sie bspw. mit GP20-BR aus der Gruppe der Sprachbewussten teilt, zeigt zudem, dass auch Überschneidungen zwischen den Gruppen existieren und die gebildeten Sprecher-/Hörertypen aufgrund der vielfältigen, individuellen Profile der Gewährspersonen nur eine grobe Gruppierung darstellen können. Festhalten lässt sich jedoch für den zweiten Sprecher-/Hörertyp, dass bei einem Teil der Gewährspersonen ein Korrelation zwischen nicht-perzipierten Varianten und der Verwendung dieser besteht sowie, bei einem anderen Teil, eine Fokussierung bestimmter sprachlicher Ebenen bei der Sprachwahrnehmung und-reflexion vorgenommen wird.

Zuletzt soll die Gruppe der Durchschnittsshörer exemplarisch betrachtet werden.

\subsection{Die Durchschnittshörer}

In die Gruppe der Durchschnittshörer fallen Gewährspersonen, die mehr als die Hälfte der Stimuli im Salienztest wahrgenommen haben, sich jedoch weder durch die Perzeption wenig salienter Merkmale wie der Typ der Sprachbewussten, noch dadurch auszeichnen, dass sie wie die Regionsverwachsenen eigentlich saliente phonetisch-phonologische Merkmale nicht perzipieren. Innerhalb der Gruppe lassen sich die älteren und die jüngeren Sprecher dahingehend voneinander unterscheiden, dass die jüngeren extrem wenige Merkmale auf der morpho-syntaktischen Ebene perzipieren, da ihnen anscheinend das Wissen über regionale bzw. standardabweichende grammatikalische Formen fehlt. Speziell bei den älteren Gewährspersonen ist dagegen zu beobachten, dass sich die metasprachliche Thematisierung regionaler Sprache weniger auf konkrete Merkmale, als auf allgemeine Sprachschichtungen in der eigenen Stadt richtet und somit hier, ähnlich wie bei den Sprachvarianztoleranten, die einschränkende Fokussierung auf bestimmte Sprachbereiche das Perzeptionsverhalten beeinflusst. Die Gewährspersonen des dritten Typs können zudem zwar als „Durchschnittshörer“, jedoch keinesfalls auch als „Durchschnittsprecher“ bezeichnet werden, da, wie auch bei den anderen Gruppen, die Sprecher auf einem Kontinuum der Standardferne bzw. -nähe auf unterschiedlichen Bereichen der Skala zu verorten sind. Zunächst soll, als Beispiel für eine Gewährsperson aus der jüngeren Gruppe, das Profil von GP12-HH auszugsweise vorgestellt werden.

\footnotetext{
${ }^{41}$ Eine gewisse Toleranz in Hinblick auf sprachliche Phänomene äußert die GP auch im Rahmen des Salienztests, in dessen Vorfeld sie angibt, ,niemanden im Sprechen korrigieren“ zu wollen, da sie nun, da sie nicht mehr im Beruf sei, „ein zweites Herz“ hätte.
} 


\begin{tabular}{|c|c|c|c|c|c|}
\hline \multicolumn{6}{|l|}{ GP12-HH (18/33) } \\
\hline & Perzeption & Wissen & $\begin{array}{c}\text { SdV pro- } \\
\text { zentual }\end{array}$ & $\begin{array}{c}\text { SV } \\
\text { absolut }\end{array}$ & $\begin{array}{c}\text { SdV ab- } \\
\text { solut }\end{array}$ \\
\hline Spirantisierung -urg & + & & $0,0 \%$ & 14 & 0 \\
\hline Alveolares [s] & + & + & $0,0 \%$ & 116 & 0 \\
\hline Diphthongierung /o:/ & & & $0,0 \%$ & 355 & 0 \\
\hline Assimilation /nd/ & & & $13,2 \%$ & 66 & 10 \\
\hline Spirantisierung /g/ &,++ & & $11,1 \%$ & 48 & 6 \\
\hline$/ \varepsilon: /$ als [e:] & & & $88,9 \%$ & 4 & 32 \\
\hline Diphthongierung /e:/ & + & & $0,0 \%$ & 189 & 0 \\
\hline Rundung /I/ zu [Y] & & & $5,1 \%$ & 356 & $19(+1)^{42}$ \\
\hline Ausfall Affrikatenkomp. & & & $100,0 \%$ & 0 & 3 \\
\hline Lenisierung /t/ & + & & $17,5 \%$ & 113 & 24 \\
\hline Vokalkürzung /a:/ & & & $33,3 \%$ & 2 & 1 \\
\hline Velarisierung /a:/ & + & & $0,0 \%$ & 395 & 0 \\
\hline Vokalisierung /r/ & & & $0,0 \%$ & 231 & 0 \\
\hline$/ \mathrm{t} /$-Elision & & & $90,8 \%$ & 7 & 69 \\
\hline Lenisierung / $\mathrm{k} /$ & + & & $5,4 \%$ & 35 & 2 \\
\hline Lenisierung $/ \mathrm{p} /$ & + & & $15,4 \%$ & 11 & 2 \\
\hline wie statt als & & & $0,0 \%$ & 5 & 0 \\
\hline Trennung ProAdv & & & $0,0 \%$ & 4 & 0 \\
\hline nach statt $z u$ & + & & & & \\
\hline Plural mit -en & + & & & & \\
\hline \multicolumn{6}{|l|}{ Akk. statt Dat. } \\
\hline \multicolumn{6}{|l|}{ Dat. statt Akk. } \\
\hline Lexem $u m z u$ & + & & & & \\
\hline
\end{tabular}

Tabelle 6: Sprecher-Hörerprofil von GP12-HH (Ausschnitt)

GP12-HH ist männlich und wurde 1985 in Hamburg geboren. Die Gewährsperson besitzt Abitur, hat Physik studiert und arbeitet als wissenschaftlicher Mitarbeiter im naturwissenschaftlichen Bereich. Er nimmt im Salienztest 18 Stimuli wahr, wobei auffällig ist, dass im morpho-syntaktischen Bereich, wie auch bei den anderen beiden jüngeren Befragten in der Gruppe der Durchschnittshörer, ${ }^{43}$ lediglich nach Müllers und Stücken als abweichend perzipiert werden. ${ }^{44}$ Bis auf die Spirantisierungen in Wech und gesacht nimmt GP12-HH keine mäßig oder gering salienten Merkmale wahr. Dass er genau diese Varianten als auffällig beurteilt, kann in Bezug zu den objektiven Sprachdaten gesetzt werden, da GP12-HH kaum spirantisierte Formen verwendet und für dieses Phänomen im Korpus einen der geringsten Realisierungswerte von nur $11,1 \%$ aufweist. ${ }^{45}$ Weitere regionalsprachliche Belege finden sich lediglich bei einer leichten Tendenz, /t/ zu lenisieren. Allgemein ist GP12-HH also als eher

42 Der Wert in Klammern bezieht sich auf einen Beleg für die Senkung zu [ $\varepsilon]$.

43 Es handelt sich um GP13-HH und GP15-HH, die genauer in Hettler 2015 thematisiert werden.

44 Die anderen beiden jüngeren Gewährspersonen in dieser Gruppe weisen dasselbe Muster auf.

${ }^{45}$ Spirantisierte Formen treten zudem nur lexemgebunden in Kombinationen mit dem Verb „kriegen“ auf (,,kriecht“", ,mitkriecht"). 
standardnaher Sprecher einzuordnen. Ähnlich wie GP11-HH aus der Gruppe der Regionsverwachsenen bezeichnet GP12-HH das Hochdeutsch in Hamburg als ,klares Hochdeutsch nach den eigentlichen Regeln“, wenngleich er im Laufe des Interviews als (einziges) Merkmal des Hamburgischen das „spitze s“ anführt. Zudem werden Phänomene der Grammatik wie der Ausfall von Präpositionen (,,ich geh mal Fitness“") thematisiert, wobei diese nicht als Teil einer regionalen Umgangssprache definiert, sondern als „Hamburger Slang“ charakterisiert werden, der in der Beschreibung an eine Form des Kiezdeutschen, wie sie bspw. Wiese thematisiert, erinnert. ${ }^{46}$ Regionale Charakteristika der eigenen Sprache nennt GP12-HH dementsprechend nicht und weist nur auf Faktoren wie ,undeutliches“ Sprechen hin. Obwohl er schon von anderen als Hamburger identifiziert wurde, sieht er die Gründe dafür genau wie GP11-HH darin, dass er reines Hochdeutsch sprechen würde.

Anders als GP11-HH gibt GP12-HH jedoch an, darauf zu achten, wie andere sprechen (so parodiert bzw. imitiert er auch gerne Dialekte) und äußert, dass ,gutes Deutsch“ und die Grammatik zumindest beruflich wichtig wären. Da er dennoch nur wenige standardabweichende Varianten der morpho-syntaktischen Ebene wahrnimmt, drängt sich die Vermutung auf, dass die geringe Perzeption morpho-syntaktischer Varianten bei den jüngeren Hamburger Gewährspersonen nicht nur mit dem individuellen Normhorizont bzw. einer gewissen Toleranz in Bezug auf grammatikalische Phänomene zu tun hat, sondern dass schlicht das Wissen über bestimmte regionalsprachliche, morpho-syntaktische Varianten fehlt, da dieses z. B. in der Schule nicht vermittelt wurden. Anders sieht dies bei den über fünfundsechzigjährigen Sprechern in der Gruppe der Durchschnittshörer aus, die den jüngeren Gewährspersonen dieser Gruppe zwar in der Anzahl perzipierter Merkmale ähneln, jedoch nicht in Bezug auf die Art dieser. Als exemplarisches Beispiel für einen älteren Sprecher mit einem durchschnittlichen Perzeptionsprofil und einem deutlichem regionalsprachlichen Anteil im Sprachmuster wird im Folgenden zuletzt das Profil von GP5-HH betrachtet.

\begin{tabular}{|c|c|c|c|c|c|}
\hline \\
\hline GP5-HH (19/33) & Perzeption & Wissen & $\begin{array}{c}\text { SdV pro- } \\
\text { zentual }\end{array}$ & $\begin{array}{c}\text { SV } \\
\text { absolut }\end{array}$ & $\begin{array}{c}\text { SdV } \\
\text { absolut }\end{array}$ \\
\hline Spirantisierung -urg & & & $\begin{array}{c}89,5 \% / \\
10,5 \% 47 \\
\end{array}$ & 0 & $17 ; 2$ \\
\hline Alveolares [s] & + & + & $8,6 \%$ & 128 & 12 \\
\hline Diphthongierung /o:/ & & & $5,2 \%$ & 398 & 22 \\
\hline Assimilation /nd/ & & & $10,8 \%$ & 74 & 9 \\
\hline Spirantisierung /g/ & & & $64,4 \%$ & 26 & 47 \\
\hline$/ \varepsilon: /$ als $[\varepsilon]$ & & & $91,7 \%$ & 3 & 33 \\
\hline Diphthongierung /e:/ & + & & $3,1 \%$ & 221 & 7 \\
\hline Rundung /I/ zu [Y] & & & $0,6 \%$ & 335 & 2 \\
\hline Ausfall Affrikatenkomp. & & & $87,5 \%$ & 1 & 7 \\
\hline Lenisierung /t/ & + & & $42,1 \%$ & 110 & 80 \\
\hline Vokalkürzung /a:/ & & & $50,0 \%$ & 3 & 3 \\
\hline
\end{tabular}

46 Cf. Wiese 2012.

47 Hier liegen sowohl Werte für die Realisierung von Hambuich als auch von Hamburch (ohne Vokalisierung von $/ \mathrm{r} /$ ) vor. 


\begin{tabular}{|l|c|c|c|c|c|}
\hline GP5-HH (19/33) & & & & & \\
\hline & Perzeption & Wissen & $\begin{array}{c}\text { SdV pro- } \\
\text { zentual }\end{array}$ & $\begin{array}{c}\text { SV } \\
\text { absolut }\end{array}$ & $\begin{array}{c}\text { SdV } \\
\text { absolut }\end{array}$ \\
\hline Velarisierung /a:/ & + & & $13,5 \%$ & 494 & 77 \\
\hline Vokalisierung/r/ & & & $0,9 \%$ & 232 & 2 \\
\hline /t/-Elision & & & $82,4 \%$ & 12 & 56 \\
\hline Lenisierung /k/ & + & & $10,0 \%$ & 36 & 4 \\
\hline Lenisierung/p/ & + & & $9,1 \%$ & 20 & 2 \\
\hline wie statt als & + & & $0,0 \%$ & 0 & 0 \\
\hline Trennung ProAdv & ++ & & $11,1 \%$ & 8 & 1 \\
\hline nach statt $z u$ & + & & & & \\
\hline Plural mit -en & + & & & & \\
\hline Akk. statt Dat. & + & & & & \\
\hline Dat. statt Akk. & + & & & & \\
\hline Lexem umzu & + & & & & \\
\hline
\end{tabular}

Tabelle 7: Sprecher-Hörerprofil von GP5-HH (Ausschnitt)

GP5-HH ist männlich und wurde 1940 in Hamburg geboren. In Hamburg hat er bis 1995 gelebt und wohnt nun in Münsterdorf bei Itzehoe und an der Eider. GP5-HH besitzt Abitur, hat Chemie studiert und u. a. in Hamburg am Seminar für Lehrerfortbildung gearbeitet. Laut Eigenaussage spricht die Gewährsperson auch Niederdeutsch. ${ }^{48}$ Im Salienztest nimmt GP5$\mathrm{HH}$ jene Stimuli, die im Rahmen der quantitativen Untersuchungen als deutlich salient befunden wurden (z. B. S-törtebeker, Joahren), wahr, jedoch kein wenig salientes Merkmal der phonetisch-phonologischen Ebene. Auffällig ist, dass die GP weder den Stimulus Hambuich noch die anderen Stimuli, die Spirantisierungen aufweisen, als auffällig kennzeichnet. Im morpho-syntaktischen Bereich dagegen hört die GP alle standarddivergenten Merkmale. Bis auf das „S-tolpern übern sp-itzen S-tein“ und eine besondere Aussprache des „a“ („Jaa“) kann GP5-HH keinen expliziten Einzelmerkmale des Hamburgischen bzw. Bremischen nennen, auch wenn sie sich bewusst ist, dass es diese gibt. Sie spricht das Problem der Abrufbarkeit und Thematisierung sprachlichen Wissens explizit im Interview an:

Es sind bestimmte Sprachmerkmoale [...] im Gehirn abgespeichert. Typisch Hamburgisch. Typisch Bremisch. Und im Grunde, bei mir isses jedenfalls so, vergleicht man, wird also verglichen, also so'n Abgleich da, wo man sacht: So, also da is das Muster Hambuich [...] abber solche typischen Merkmoale, die falln mir im Moment nich ein für Hambuich. Das kann ich nich sagn, gut.

(Zitat aus dem Interview von GP5-HH)

Auch Merkmale der eigenen Sprache kann die Gewährsperson bis auf die Annahme, dass er das „st [...], ohne es zu merken,“ benutzt, nicht nennen. Die eigene Sprache ist aber norddeutsch gefärbt: „Also man hört wohl, wo ich herkomm. Und das is mehr Hamburgisch,

\footnotetext{
48 Die Möglichkeit, Niederdeutsch zu sprechen, besteht vorrangig im Kontakt mit Bekannten an der Eider. Nach der Kategorisierung von Jürgens (2015) wäre GP5-HH somit wohl am ehesten als „Gelegenheitssprecher“ einzuordnen, wobei es sich bei ihm nicht um zufällig auftretende Möglichkeiten zur Nutzung der Sprache handelt, sondern der Befragte einen festen Kreis an Personen besitzt, mit denen er Niederdeutsch sprechen kann, was ihn auch in die Nähe der „Alltagssprecher“ rückt (cf. Jürgens 2015: 175-188).
}

ISSN 1615-3014 
würd ich sagen.“ GP5-HH ist dementsprechend auch schon häufiger als „Fischköpp“ identifiziert worden und sagt zur regionalen Färbung seiner Sprache: „Die Muttersprache kann man nich verleugnen, das is ne Preegung, die bleibt. Man selber hört es gar nich.[...] aber man fällt auf damit.“ Die Einschätzung der Gewährsperson, regional zu sprechen, wird durch die Ergebnisse der Type-Token-Analysen bestätigt. Auffällig ist der Befund bei den Spirantisierungen - so realisiert der Befragte die Spirantisierung von /g/ + Vokalisierung von /r/ durchgehend bei allen Namen, die auf -burg enden, wie auch in Harbuich oder Mecklenbuich, und auch der allgemeine Anteil an Spirantisierungen ist hoch. Zudem ist der Ausfall der Affrikatenkomponente bei dem Sprecher relativ durchgängig. Der Anteil der Lenisierungen ist beim /t/ mit 42,1\% besonders hoch, während er bei / $/$ oder /p/ geringer ist. Zugleich sind viele Belege (77) für velarisiertes /a:/ zu finden sowie, allerdings in weniger starkem Maße, für die Diphthongierung von /o:/. Auch finden sich Belege für den Gebrauch des alveolaren [s]. Anders als bei anderen Sprechern ist jedoch kein lexemgebundenes Muster zu erkennen, ${ }^{49}$ was auch der Selbsteinschätzung der Gewährsperson, die Variante „ohne es zu merken“ zu verwenden, entspricht. Abgesehen von den Lenisierungen und dem alveolaren [s] wird ein Großteil der Merkmale, die von GP5-HH in den Sprachproben realisiert werden, nicht gehört. Besonders auffällig ist die Korrelation bei den Spirantisierungen die, wie thematisiert, nicht perzipiert werden. Obwohl der Befragte, wie auch die Regionsverwachsenen, viele Merkmale der eigenen Sprache nicht bemerkt, hört er dennoch auch selbst verwendete saliente Merkmale wie die Lenisierung von /t/ in Reddungsfahrzeuge. Im Interview präsentiert sich GP5-HH zudem als Sprecher, der sich verschiedener Abstufungen der Sprache in Hamburg allgemein und auch seiner eigenen Sprache zwischen den Polen Niederdeutsch und Standardsprache bewusst ist. Die Sprache in Hamburg wird als „Norddeutsch“ bezeichnet, daneben nennt er noch den Begriff „Missingsch“, der in dem Stadtteil, in dem die GP aufgewachsen ist (Wandsbek), gesprochen wurde. ${ }^{50}$ Auch ist sich die Gewährsperson der diachronen Entwicklung von Missingsch bewusst: „Ich würd mal sagen der Anteil des Niederdeutschen am Missingsch hat sich von meiner Zeit bis heude in Anführungs-trichen zum Nachteil des Niederdeutschen verschoben." Zudem wird auch der eigene Niederdeutschgebrauch kritisch thematisiert, da sich GP5-HH bewusst ist, dass er aufgrund lexikalischer Lücken das Niederdeutsche mit dem Hochdeutschen mischt:

Man man kann [bei mir, Y.H.] nich sagen: Hamburger Platt. [...] Ich weiß nur, dass mich die Leute $[\ldots]$ verstäihen [...] und auch mit mir sich 'ne halbe Stunde sich unterhalten. Trotzdem, manchmal weiß ich bestümmte Begriffe nich und dann geh ich auf Hochdeutsch und da fällt es dann natürlich auf.“

(Zitat von GP5-HH)

\footnotetext{
${ }^{49}$ So gibt u. a. der ansonsten sehr standardnahe Sprecher GP20-BR an, alveolares [s] lediglich in gewissen Lexemen wie Ortsbezeichnungen zu verwenden. Diese Einschätzung bestätigt sich auch im Rahmen der objektiven Daten.

${ }^{50} \mathrm{Da}$ es heutzutage zu gut wie keine Sprecher mehr gibt, die ausschließlich Niederdeutsch als L1 aufweisen, ist Missingsch nach Möhn aus synchroner Sicht als eine Varietät des Hochdeutschen auf niederdeutschem Substrat einzuordnen, wobei es „einer gewissen Dichte von Erscheinungen im selben Text [bedarf], um ihn als ,missingsch“ auszumachen“ und es sich ansonsten ,[...] eher um einzelne ,Fehler' oder Abweichungen [handelt], die in der Gegenwart als Merkmale des Substandards oder auch noch als umgangssprachlich klassifiziert werden“ (Möhn 2004: 127).
} 
Konkrete Beispiele hierfür kann GP5-HH, wie auch bei der Frage nach spezifischen Merkmalen des Hamburgischen, nicht nennen. Festzuhalten ist, dass bei GP5-HH zwar, ähnlich wie bei den Sprachbewussten, eine ausgeprägte Sprachbewusstheit und auch ein Interesse an Sprache konstatiert werden kann, sich die Thematisierung metasprachlichen Wissens jedoch mehr auf Varietäten und weniger auf Varianten bezieht. Sein Perzeptionsmuster ist zudem nur als durchschnittlich zu bezeichnen, wobei selbst verwendete Varianten, ähnlich wie bei den Regionsverwachsenen, tendentiell eher nicht wahrgenommen werden.

Zusammenfassend definiert sich die Gruppe der Durchschnittshörer also vornehmlich dadurch, dass sie kein „besonderes“ Perzeptionsverhalten zeigt, sondern erwartbare saliente Merkmale wahrgenommen werden, während nicht saliente Varianten wie die /t/-Elision nicht gehört werden. In Bezug auf das Sprachwissen oder das Normverständnis kann jedoch keine Einheitlichkeit festgestellt werden. Auch zeichnet sich innerhalb der Gruppe deutlich die Untergruppe der drei jüngeren Hamburger GP ab, die speziell im morpho-syntaktischen Bereich weniger Merkmale als die anderen Hörer der Gruppe wahrnehmen.

\section{$6 \quad$ Schluss}

Zusammenfassend zeigt die vorliegende Studie durch die Verknüpfung von subjektiven und objektiven Daten, dass die Wahrnehmung von standardabweichenden Varianten als auffällig das Resultat eines merkmalsindividuellen, komplexen Zusammenspiels verschiedener Parameter ist. Als wichtige Faktoren, die die Wahrnehmung von Merkmalen bedingen, lassen sich u. a. eine gut ausgeprägte Sprachbewusstheit, die Fähigkeit, metasprachliches Wissen auch verbal zu thematisieren sowie, damit einhergehend, ein allgemeines Interesse an Sprache bzw. Regionalsprache konstatieren. Auch ein besonders hohes Normempfinden (z. B. studienoder berufsbedingt) kann beeinflussend wirken. Der indivuelle Gebrauch regionaler Formen kann jedoch je nach Sprecher unterschiedlich mit der Perzeption dieser korrelieren. So zeigt sich bei den vorgestellten Sprecher-/Hörertypen, dass sich innerhalb jeder Gruppe sowohl standardnahe Sprecher finden lassen als auch Sprecher, die einen relativ hohen Anteil regionaler Varianten in ihrem Sprachgebrauch aufweisen.

Dass sprecher- bzw. hörerindividuelle Faktoren wie das Normverständnis und die Sprachbewusstheit stark die Sprachwahrnehmung beeinflussen, bedeutet auch, dass bei Erhebungen zur Salienz nicht nur quantitativ, sondern auch verstärkt qualitativ gearbeitet werden sollte. Dies gilt umso mehr, als anzunehmen ist, dass noch weitere hörer- bzw. sprecherindividuelle Faktoren, die im Rahmen der Interviews nicht thematisiert wurden, Einfluss auf das metasprachliche Wissen und die Sprachwahrnehmung von Sprechern haben und in weiteren Studien untersucht werden müssen.

\section{Literaturverzeichnis}

Anders, Christina Ada (2010a): Wahrnehmungsdialektologie. Das Obersächsische im Alltagsverständnis von Laien. Berlin/New York: de Gruyter. (= Linguistik - Impulse und Tendenzen 36).

Anders, Christina Ada (2010b): „Die wahrnehmungsdialektologische Rekodierung von laienlinguistischem Alltagswissen“. In: Anders, Christina Ada/Hundt, Markus/Lasch, Alexan- 
der (eds.): Perceptual Dialectology. Neue Wege der Dialektologie. Berlin/New York, de Gruyter: 67-89. (= Linguistik-Impulse und Tendenzen 38).

Berend, Nina (2005): „Regionale Gebrauchsstandards - Gibt es sie und wie kann man sie beschreiben?“. In: Eichinger, Ludwig Maximilian/Kallmeyer, Werner (eds.): Standardvariation. Wie viel Variation verträgt die deutsche Sprache? Berlin/New York, de Gruyter: 143-170.

Bieberstedt, Andreas/Ruge, Jürgen/Schröder, Ingrid (2011): Hamburgisch - Sprachkontakt und Sprachvariation im städtischen Raum. Hamburger Transkriptionskonventionen. www.docplayer.org/43778478-Hamburger-transkriptionskonventionen.html [28.4.2016].

Bieberstedt, Andreas/Ruge, Jürgen/Schröder, Ingrid (2016): „Hamburger Transkriptionskonventionen“. In: Bieberstedt, Andreas/Ruge, Jürgen/Schröder, Ingrid (eds.): Hamburgisch. Struktur, Gebrauch, Wahrnehmung der Regionalsprache im urbanen Raum. Frankfurt a. M., Lang: 421-428. (= Sprache in der Gesellschaft 34).

DeKeyser, Robert M. (2009): "Cognitive-psychological processes in second language learning“. In: Long, Michael H./Doughty, Catherine J. (eds.): The handbook of language teaching. Malden, Wiley-Blackwell: 119-138.

Edmondson, Willis James/House, Juliane (1997): „Language Awareness. Zur Einführung in den Themenschwerpunkt". Fremdsprachen Lehren und Lernen 26: 3-8.

Elmentaler, Michael et al. (2006): „Sprachvariation in Norddeutschland. Ein Projekt zur Analyse des sprachlichen Wandels in Norddeutschland“. Osnabrücker Beiträge zur Sprachtheorie 71: 159-178.

Elmentaler, Michael/Gessinger, Joachim/Wirrer, Jan (2010): „Qualitative und quantitative Verfahren in der Ethnodialektologie von Salienz“. In: Anders, Christina Ada/Hundt, Markus/Lasch, Alexander (eds.): Perceptual Dialectology. Neue Wege der Dialektologie. Berlin/New York, de Gruyter: 111-150. (=Linguistik - Impulse und Tendenzen 38).

Fehling, Sylvia (2010): Language Awareness und bilingualer Unterricht. Eine komparative Studie. 2., überarbeitete Auflage. Frankfurt a. M.: Lang. (=Language Culture Literacy 1).

Gessinger, Joachim/Butterworth, Judith (2015): „Salienz als dynamisches interaktionales Konstrukt. Theoretische und methodische Aspekte“. In: Elmentaler, Michael/Hundt, Markus/Schmidt, Jürgen Erich (eds.): Deutsche Dialekte. Konzepte, Probleme, Handlungsfelder. Akten des 4. Kongresses der Internationalen Gesellschaft für Dialektologie des Deutschen (IGDD). Stuttgart, Steiner: 259-295.

Glauninger, Manfred Michael (2014): „Salienz und System“. Linguistik online 66: 21-29.

Gnutzmann, Claus (2012): „Kennen und Können: Hängt das zusammen?“. In: BurwitzMelzer, Eva/Königs, Frank Gerhard/Krumm, Hans-Jürgen (eds.): Sprachenbewusstheit im Fremdsprachenunterricht. Arbeitspapiere der 32. Frühjahrskonferenz zur Erforschung des Fremdsprachenunterrichts. Tübingen, Narr: 40-47. (= Giessener Beiträge zur Fremdsprachendidaktik).

Gombert, Jean Émile (1992): Metalinguistic development. Chicago: University of Chicago Press.

Green, Peter S./Hecht, Karlheinz (1993): “Language awareness of German pupils". Language awareness 2/3: 125-142. 
Häcki Buhofer, Annelies (2002): „Steuert Sprachbewusstheit den eigenen Sprachgebrauch? Überlegungen zum Zusammenhang an Beispielen aus der deutschen Schweiz“. Der Deutschunterricht 3/54: 19-30.

Hettler, Yvonne (2014): ,Salienz, Bewertung und Realisierung regionaler Merkmale in Norddeutschland“. Linguistik online 66: 71-90.

Hettler, Yvonne (2015): Salienz, Bewertung und Realisierung regionaler Sprachmerkmale in Bremen und Hamburg. Hamburg: Dissertation.

Hettler, Yvonne (2016): „,Die Bremer sprechen natürlich immer dieses ,e“ so komisch“. Laienlinguistische Selbst- und Fremdwahrnehmung in Bremen und Hamburg“. In: Bieberstedt, Andreas/Ruge, Jürgen/Schröder, Ingrid (eds.): Hamburgisch. Struktur, Gebrauch, Wahrnehmung der Regionalsprache im urbanen Raum. Frankfurt a. M., Lang: 171-215. (= Sprache in der Gesellschaft 34).

Hinneberg, Sabrina (2005): Sprachbewusstheit und Aphasie: eine Untersuchung metasprachlicher Fähigkeiten. Hamburg: Kovač.

Höder, Steffen (2003): „Wi Hamburger schnackt maal so, maal so. Hochdeutsch und Niederdeutsch in den Äußerungen bilingualer Sprecher". Korrespondenzblatt des Vereins für niederdeutsche Sprachforschung 110: 47-56.

Hug, Michael (2007): „Sprachbewusstheit/Sprachbewusstsein - the state of the art“. In: Hug, Michael/Siebert-Ott, Gesa (eds.): Sprachbewusstsein und Mehrsprachigkeit. Hohengehren, Schneider: 10-31. (= Diskussionsforum Deutsch 26).

Ivo, Hubert/Neuland, Eva (1991): „Grammatisches Wissen. Skizze einer empirischen Untersuchung über Art, Umfang und Verteilung grammatischen Wissens (in der Bundesrepublik)“. Diskussion Deutsch 121: 437-493.

Johnstone, Barbara (2007): "Linking identity and dialect through stancetaking". In: Englebretson, Robert (ed.): Stancetaking in discourse. Subjectivity, evaluation, interaction. Amsterdam etc., Benjamins: 49-68.

Jürgens, Carolin (2015): Niederdeutsch im Wandel. Sprachgebrauchswandel und Sprachwahrnehmung in Hamburg. Hildesheim: Olms. (= Deutsche Dialektgeographie 119).

Kerswill, Paul/Williams, Ann: “'Salience' as an explanatory factor in language change; evidence from dialect levelling in urban England”. In: Jones, Mari C./Esch, Edith (eds.): Language Change. The Interplay of Internal, External and Extra-Linguistic Factors. Berlin/New York, de Gruyter: 81-110. (= Contributions to the society of language 86).

Keuch, Sarah/Wirrer, Jan (2014): „,Da saßen zwei so 'ne alte Friedrichskooger neben mir auf der Bank. Da hab ich mir gedacht: Das hast du lange nicht gehört, also wirklich so extrem breites und tiefes Plattdeutsch'. Laikale metasprachliche Wissensbestände und Sprechertypologie“. In: Cuonz, Christina/Studler, Rebekka (eds.): Sprechen über Sprache. Perspektiven und neue Methoden der Spracheinstellungsforschung. Tübingen, Stauffenburg: 65105.

Kiesewalter, Carolin (2011): „Zur Salienz remanenter Merkmale des Neuhessischen“. In: Ganswindt, Brigitte/Purschke, Christoph (eds.): Perspektiven der Variationslinguistik. Beiträge aus dem Forum Sprachvariation. Hildesheim, Olms: 335-369. (= Germanistische Linguistik 216/217).

Kiesewalter, Carolin (2014): „Salienz und Pertinenz. Zur subjektiven Dialektalität remanenter Regionalismen im Mittelbairischen“. Linguistik online 66: 111-134. 
Labov, William (1966): The social stratification of English in New York City. Washington: Center for Applied Linguistics.

Lehr, Andrea (2002): Sprachbezogenes Wissen in der Lebenswelt des Alltags. Tübingen: Niemeyer. (= Reihe Germanistische Linguistik 236).

Lenz, Alexandra N. (2010): „Zum Salienzbegriff und zum Nachweis salienter Merkmale“. In: Anders, Christina Ada/Hundt, Markus/Lasch, Alexander (eds.): Perceptual Dialectology. Neue Wege der Dialektologie. Berlin/New York, de Gruyter: 89-110. (=Linguistik - Impulse und Tendenzen 38).

Lorenz, Cornelia (2014): „Salienz unter Einheimischen und Zugezogenen - Ein empirischer Vergleich“. Linguistik online 66: 135-146.

Mihm, Arend (1985): „Prestige und Stigma des Substandards, Zur Bewertung des Ruhrdeutschen im Ruhrgebiet". In: Mihm, Arend (ed.): Sprache an Rhein und Ruhr: dialektologische und soziolinguistische Studien zur sprachlichen Situation im Rhein-Ruhr-Gebiet und ihrer Geschichte. Stuttgart, Steiner: 163-194. (= Zeitschrift für Dialektologie und Linguistik Beiheft 50).

Möhn, Dieter (2004): „Missingsch“. In: Munske, Horst Haider (ed.): Deutsch im Kontakt mit germanischen Sprachen. Tübingen, Niemeyer: 119-140.

Peter, Klaus (2011): Textbewertungen. Eine empirische Untersuchung zu Sprachbewusstheit und Spracheinstellungen. Tübingen: Stauffenburg.

Purschke, Christoph (2011): Regionalsprachlichkeit und Hörerurteil. Grundzüge einer perzeptiven Variationslinguistik. Stuttgart: Steiner. (=Zeitschrift für Dialektologie und Linguistik Beiheft 149).

Purschke, Christoph (2014): „'I remember it like it was interesting.' Zur Theorie von Salienz und Pertinenz". Linguistik online 66: 31-50.

Pratt, Christopher/Grieve, Robert (1984): "The development of Metalinguistic Awareness: An Introduction". In: Tunmer, William E./Pratt, Christopher/Herriman, Michael L. (eds.): Metalinguistic awareness in children. Theory, research and implications. Berlin etc., Springer: 2-35.

Preston, Dennis (2002): "Language with an Attitude”. In: Chambers, Jack K./Trudgill, Peter/Schilling-Estes, Natalie (eds.): The Handbook of Language Variation and Change. Malden, Wiley-Blackwell: 40-66.

Rácz, Péter (2012): "Operationalising Salience. Definitive Article Reduction in the North of England". English Language and Linguistics 16: 57-79.

Scherfer, Peter (1983): Untersuchungen zum Sprachbewusstsein der Patois-Sprecher in der Franche-Comté. Tübingen: Narr.

Schirmunski, Viktor (1928/1929): „Die schwäbischen Mundarten in Transkaukasien und Südukraine“. Teuthonista 5: 38-60 und 157-171.

Schmidt, Richard (1994): "Deconstructing consciousness in search of useful definitions for applied linguistics". AILA Review 11: 11-26.

Trudgill, Peter (1986): Dialects in contact. Oxford: Blackwell. (=Language in society 10).

Wehr, Silke (2001): Was wissen Kinder über Sprache? Die Bedeutung von Meta-Sprache für den Erwerb von Schrift- und Lautsprache. Bern: Haupt.

Wiese, Heike (2012): Kiezdeutsch. Ein neuer Dialekt entsteht. München: Beck.

Wolff, Dieter (1993): „Sprachbewusstheit und die Begegnung mit Sprachen“. Die neueren Sprachen 92/6: 510-531. 
Ziegler, Evelyn (1996): Sprachgebrauch, Sprachwissen, Sprachvariation. Eine Familienfallstudie. Frankfurt a. M.: Lang. (=Variolingua 2). 